\title{
PEGASE, an infrared interferometer to study stellar environments and low mass companions around nearby stars
}

\author{
M. Ollivier • O. Absil · F. Allard · J.-P. Berger · P. Bordé · F. Cassaing • \\ B. Chazelas - A. Chelli • O. Chesneau • V. Coudé du Foresto $\cdot$ D. Defrère • \\ P. Duchon · P. Gabor • J. Gay · E. Herwats · S. Jacquinod · P. Kern • \\ P. Kervella · J.-M. Le Duigou • A. Léger · B. Lopez • F. Malbet • \\ D. Mourard · D. Pelat • G. Perrin • Y. Rabbia • D. Rouan • \\ J.-M. Reiss • G. Rousset • F. Selsis • P. Stee • J. Surdej
}

Received: 24 January 2008 / Accepted: 10 December 2008 / Published online: 13 January 2009

(C) Springer Science + Business Media B.V. 2009

\begin{abstract}
PEGASE is a mission dedicated to the exploration of the environment (including habitable zone) of young and solar-type stars (particularly those in the DARWIN catalogue) and the observation of low mass companions around nearby stars. It is a space interferometer project composed of three free flying spacecraft, respectively featuring two $40 \mathrm{~cm}$ siderostats and a beam
\end{abstract}

M. Ollivier $(\varangle) \cdot$ P. Gabor · S. Jacquinod · A. Léger

Institut d'Astrophysique Spatiale d'Orsay, Université de Paris-Sud XI and CNRS (UMR 8617), Bâtiment 121, 91405 Orsay cedex, France

e-mail: Marc.ollivier@ias.u-psud.fr

O. Absil · D. Defrère · E. Herwats $\cdot$ J. Surdej

Université de Liège, Liège, Belgium

F. Allard

Ecole Normale Supérieure de Lyon, Lyon, France

J.-P. Berger · A. Chelli · P. Kern · F. Malbet

Laboratoire d'Astrophysique de l'Observatoire de Grenoble, Grenoble, France

P. Bordé · B. Chazelas

Institut d'Astrophysique Spatiale d'Orsay, Université de Paris-Sud, Bâtiment 121,

91405 Orsay cedex, France

F. Cassaing

Office National d'Etudes et de Recherches Aéronautiques, Paris, France

O. Chesneau · J. Gay · B. Lopez

Observatoire de la Côte d'Azur, Nice, France

O. Chesneau · D. Mourard · Y. Rabbia · P. Stee

Observatoire de la Côte d'Azur, Grasse, France 
combiner working in the visible and near infrared. It has been proposed to ESA as an answer to the first "Cosmic Vision" call for proposals, as an M mission. The concept also enables full-scale demonstration of space nulling interferometry operation for DARWIN.

Keywords Nulling interferometry $\cdot$ Low mass objects $\cdot$ Space interferometry

\section{Introduction}

Several concepts have been proposed these last years to perform direct detection and spectral analysis of terrestrial extrasolar planets (DARWIN, [11], TPF-C [4]). Preliminary studies have shown the complexity of these concepts and the idea of potential precursors appeared. PEGASE is one of these precursors. It has been first proposed to the French Space Agency $\left(\mathrm{CNES}^{1}\right)$ as an answer to a formation flying mission call for proposal. After a first selection, and a study at a 0 level concluding to the feasibility of the concept, it has been decided to submit it to ESA as an answer to the first "Cosmic Vision" call for proposals.

\section{Scientific objectives of PEGASE}

The goals of PEGASE are compact sources for which emission peaks in the 1.5-6 $\mu \mathrm{m}$ spectral range, corresponding to a temperature range from 500 to $3,000 \mathrm{~K}$ (maximum of the black body emission). In that spectral range, PEGASE will get the sensitivity of ISO but with an angular resolution from 80 to 1,000 times higher (about 0.5 mas), thanks to baselines from $40 \mathrm{~m}$ (and maybe $20 \mathrm{~m}$ ) to $500 \mathrm{~m}$. These characteristics open a new field in the study of many astrophysical sources. For each of these programs, a list of putative targets is given in the ESA original proposal.

\subsection{Disk science}

The occurrence of disks is a common feature in many astrophysical objects: AGNs, young stellar objects, main sequence stars, and rapid rotators...

\footnotetext{
${ }^{1}$ Centre National d'Etudes Spatiales.

V. Coudé du Foresto - P. Kervella · D. Pelat .

G. Perrin - D. Rouan · J.-M. Reiss · G. Rousset

Observatoire de Paris, Paris, France

P. Duchon · J.-M. Le Duigou

Centre National d'Etudes Spatiales, Toulouse, France

F. Selsis

Observatoire de Bordeaux, Bordeaux, France
} 
PEGASE, with its high dynamics range is particularly well suited to the observation of environments of bright on-axis sources.

\subsubsection{Protoplanetary disks}

It is now more and more accepted that planets form before the star reaches the main sequence and the protoplanetary disk vanishes. The study of protoplanetary disks, especially of the planet forming and wind-launching regions, allows thus understanding the planetary formation mechanisms. Several types of observations can be considered:

- Protoplanetary disk mapping: the goal is to look for gaps created by transiting planets inside the disk. This point is very important to understand the formation of planetary system and early dynamics of planets, including migration and orbital interaction. The gap, which creates a non-uniformity in the disk, can be detected by high accuracy visibility measurements. Among all types of stars and disks, gaps can particularly be observed in the case of T Tauri and FU Ori disks (Fig. 1). The gap is detected by an accurate measurement of the visibility function;

- disks around Herbig AE/BE stars: In the near infrared spectral range, in the case of massive disks, one can observe the transition between the photospheric emission of the star and the disk emission. The observation of the internal part disk where the dust grains sublimate (0.1 to $0.4 \mathrm{AU})$ is
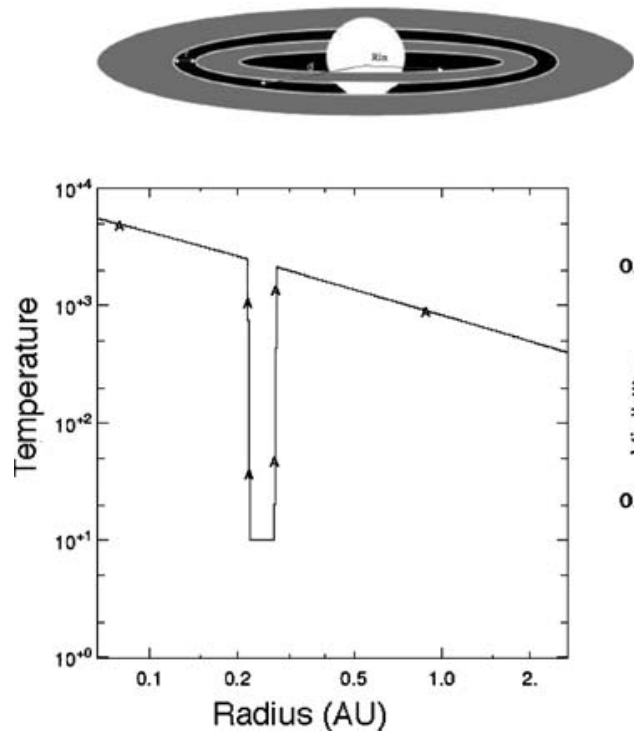

Baseline : $500 \mathrm{~m}$

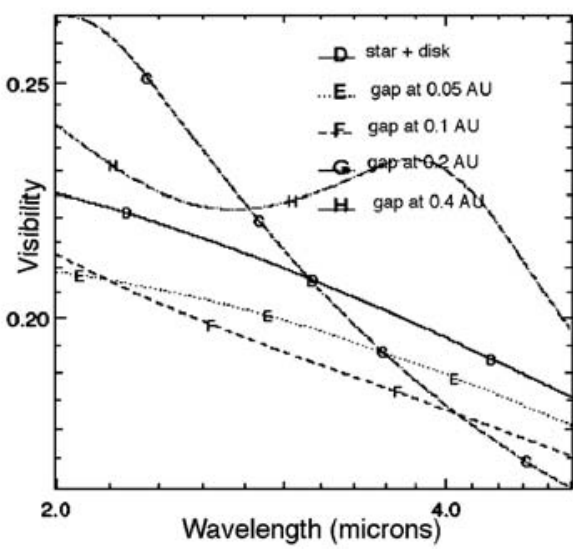

Fig. 1 Variation of the measured fringe visibility as a function of the gap radius in a FU Ori disk at $100 \mathrm{pc}$. The gap is modeled by a gap in the temperature distribution within the disk (left). The disk is considered as optically thick, and continuous until the star surface (no inner edge). The baseline is equal to $500 \mathrm{~m}$ 
thus important to constrain models. High angular resolution observations allowed by PEGASE will help mapping the dust in the disk and particularly the distribution of PAH;

- Disks around T Tauri stars: With high angular resolution and high dynamics, it is possible to constrain the size of the magnetosphere of the star and to observe the inner part of the disk, allowing the study of matter transfer from internal disk $(d<1 \mathrm{AU})$ to stellar surface.

\subsubsection{Debris disks}

The next step in understanding planetary formation is the study of debris disks. Several main sequence stars have been found by the IRAS and ISO satellites to feature excess radiation at far infrared wavelengths, which is thought to arise from short-lived dust grains in optically thin disks. This might very well be the sign of planetary activity, as the production of grains is believed (by analogy with the zodiacal cloud in our solar system) to be sustained by asteroid collisions and outgassing of comets in the first tens of $\mathrm{AU}$ and by collisions of Kuiper belt-like bodies at large distance [16].

A recent review on the topic can be found in Mann et al. [14]. Because they are much fainter than their host stars, only the brightest, most massive debris disks have been imaged so far. They have shown large-scale structures similar to our own Kuiper belt with a relative void of matter in the central regions, where planets could plausibly clear off the dust.

However, in the inner disk regions where planets could be present, warmer grains could exist, and indeed have been detected by the Spitzer Space Telescopes as photometric excesses in the $25-100 \mu \mathrm{m}$ range. However, the presence of hot grains in the first AUs of extra-solar planetary systems cannot be unequivocally determined by classical photometry, whose typical accuracy is a few percent at best in rather large fields of view.

Infrared interferometry is a more appropriate tool as it can provide sub-AU spatial resolution and spatially separate the contribution of the warm dust from that of the stellar photosphere. Indeed, near IR (K-band) thermal emission at the $\sim 1 \%$ photospheric level has already been isolated in the vicinity of Vega and tau Ceti with the CHARA/FLUOR ground-based interferometer $[1,10]$. PEGASE, providing detectability for higher contrast environments (up to $10,000: 1$ ) and at longer wavelengths, will be able to probe a much wider range of inner debris disks.

Beyond the mere photometry of warm dust, an interferometer can provide very useful information about its spatial distribution. Most of the near-IR flux is expected to be emitted by a thin ring region whose radius corresponds to the sublimation limit of the dust. Measuring the sublimation radius strongly constrains the mineralogy of the grains. This, however, requires the capability to address arbitrarily any point in the $(u, v)$ plane a feature that distinguishes PEGASE from ground-based interferometers (Fig. 2). In addition, the presence of planets in a circumstellar disk creates structures (not only gaps but also large scale structures) that can be detected by a high angular resolution, 


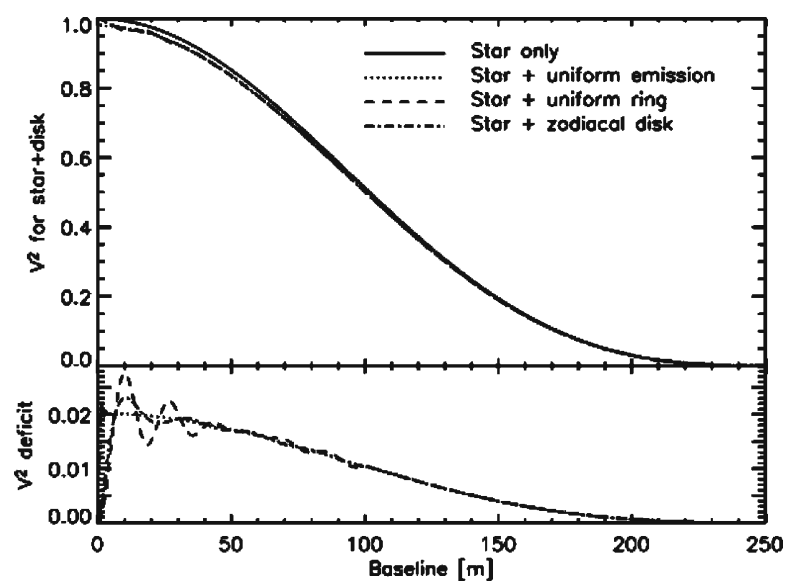

Fig. 2 Visibility of a star surrounded by circumstellar emission compared to the visibility of the star only (an A0V star at $10 \mathrm{pc}$ for this illustration), observed in the $\mathrm{K}$ band. Three different morphologies have been assumed for the circumstellar emission: a uniform emission spread over the whole field-of-view, a ring-like structure located between $0.2 \sim \mathrm{AU}$ (sublimation radius) and $0.3 \mathrm{AU}$ from the star, and a more realistic debris disk model similar to the solar zodiacal disk. All have been scaled so that their integrated brightness represents $1 \%$ of the photospheric emission

high dynamics instrument. Observation will provide us with insight into the existence and major parameters of planets themselves (mass, orbit, current location...) and existence and location of planetesimals belts, comparable to the Kuiper Belt.

\subsubsection{Exozodiacal light characterization in the context of DARWIN}

The residual glow generated by the dust particles within the habitable zone around the target star is a key parameter for the performance of a nulling interferometer, as it is likely to be the brightest component (after the star itself) of an exoplanetary system. For example in the solar system, the zodiacal light is two orders of magnitude brighter than the Earth at mid-infrared wavelengths. Warm exozodiacal cloud could thus become the main source of noise if it is more than tens times as dense as the solar zodiacal disk. Irregularities (clumps etc.) in the dust cloud may also introduce constraints on the detection capability. However, our knowledge of the dust distribution in the first few AUs around solar-type stars is currently mostly limited to the observations of the solar zodiacal disk, a sparse structure of warm silicate grains 10 to $100 \mu \mathrm{m}$ in diameter. PEGASE will have the capability of providing a statistically significant survey of the amount of exozodiacal light in the habitable zone around the DARWIN targets, and its prevalence as a function of other stellar characteristics (age, spectral type, metallicity, cold debris disk...) it will be used to streamline the input source catalogue for the Darwin survey (reducing the risk of observing sources for which exozodiacal light prevents exo-Earth detection) and optimize its mission profile before the launch. 


\subsection{Sub stellar objects: from low mass stars to exoplanets}

The main goal is to get a consistent vision of sub stellar objects formation and to understand if a limit between stars that classically form by collapse of a gas cloud and planets that form by accretion of planetesimals exists. To reach this goal it is necessary to observe gravitationally linked objects to get an accurate estimation of their mass (the mass of free floating objects cannot be determined without models based on the age and the observed flux). In the case of gravitationally linked objects, the mass is determined by the observation of the orbit and the mass of the parent star. One can then explore the diversity of these objects.

\subsubsection{Spectral characterization of hot jupiters}

A Hot Jupiter is an exoplanet with a mass comparable to Jupiter's one, orbiting in the very neighborhood of its parent star (at distance less than 0.1 a.u ie with orbital periods shorter than about 10 days). Up to now, about 50 hot Jupiters or at least hot objects (mass lower than Jupiter) are known. Present observation techniques help characterizing the mass of the objects, and elements of the atmospheric composition by spectral photometry and model fitting. Up to now, no spectrum with a resolution of several tens has been obtained. Such a spectrum would help determining the atmospheric and internal structure of the object. The understanding of the internal structure is fundamental to explain the object diversity that is observed in the mass/radius diagram where two objects with then same diameter can have a mass varying by a factor larger than 2.

Hot jupiters have no equivalent in the Solar System, and no theory had predicted their existence before their discovery. They certainly did not form where they are now, but it is still difficult to know what their presence implies on potential other planets. They are hot strongly irradiated objects $\left(T_{e f} \sim\right.$ 1,000 to $1,500 \mathrm{~K}$ ), with a rotation period certainly synchronized with the revolution period, which means that a same face of the planet is permanently irradiated leading to a complex circulation within the atmosphere. Even if all the models describe hot jupiters as giant gaseous objects, the role of aerosols and potential dust clouds, the level of thermalization between irradiated and dark hemisphere of the planet and the potential existence of winds between the hemispheres are strongly discussed. Figure 3 shows two alternative models with and without clouds leading to a strong difference in the spectral features depth. A spectral analysis of the objects with a spectral resolution of several tens in the near infrared would strongly constrain the atmospheric models.

The infrared spectroscopy in the near infrared (from 1.5 to $6 \mu \mathrm{m}$ ) has several advantages to study hot jupiters. The contrast with the central star is moderate ( $10^{5}$ to $10^{3}$ depending on wavelength). This spectral range contains spectral signatures of several gaseous compounds such as $\mathrm{CH}_{4}$ and $\mathrm{CO}$ (which depth is a tracer of cloud thickness) and mainly $\mathrm{H}_{2} \mathrm{O}$, which has an important feature at $2.6 \mu \mathrm{m}$ that is difficult to detect from ground. A photometric survey of the 
Fig. 3 Synthetic spectra of hot jupiters: top: [20], bottom: [3], same units, same scale. The Sun at the distance of 51 Peg would have a flux of about 8 Jy at $3 \mu \mathrm{m}$
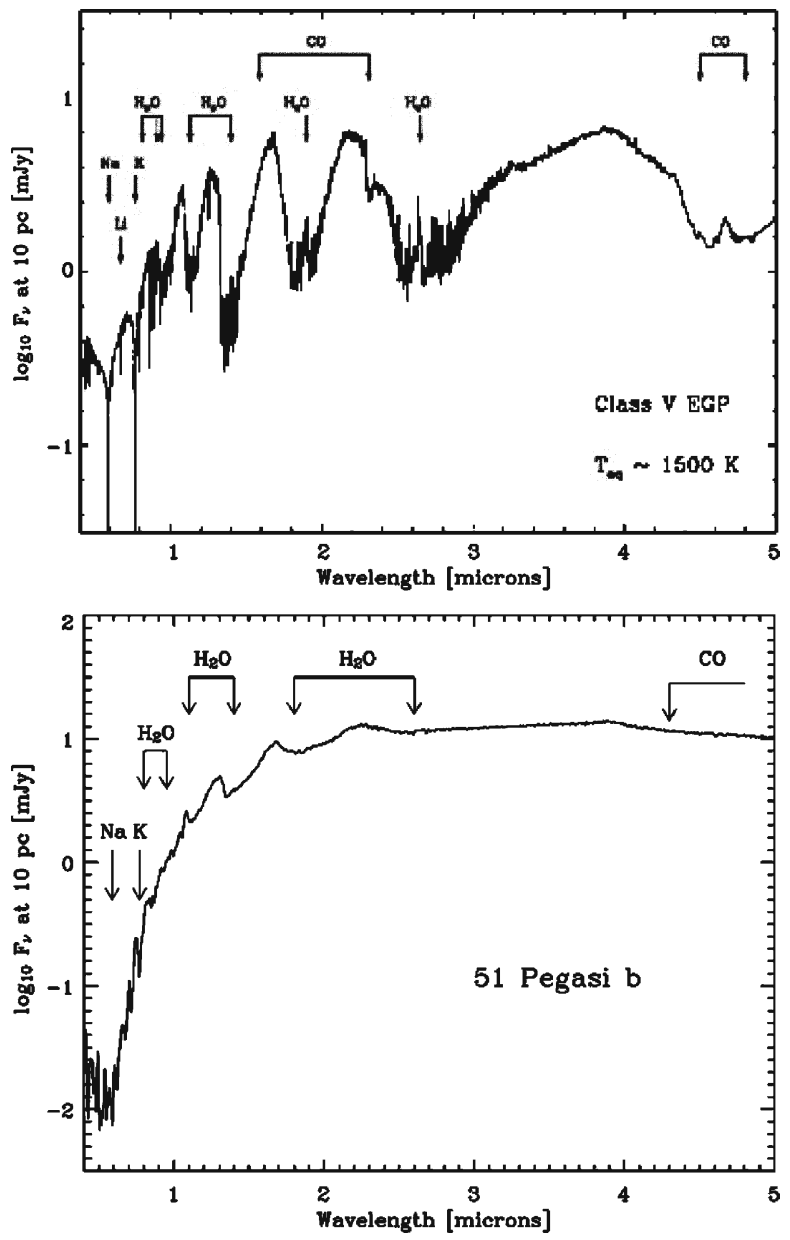

planet along its orbit allows the determination of its phase function and the level of thermalization of the high atmosphere.

At present, only transiting hot jupiters can be studied by direct or secondary transit analysis. Because of the level of parent star flux, the accuracy of the spectrum is poor. PEGASE will lead to the best spectra of such objects ever measured. In addition, the spectral range and angular resolution reached by PEGASE are unique.

\subsubsection{Internal structure and atmosphere of brown dwarfs}

Internal structure There are few observations that allow checking the physics of internal structure. In addition, modeling the atmosphere is also difficult. The information about the internal structure of red dwarfs and brown dwarfs comes from the observation of their photosphere. Observables are the effective temperature $T_{\text {ef }}(M)$ and the radius $R(M)$, which are at present not well known. 
One of the goals of internal structure modeling is to determine the relation between both quantities. From an observational point of view, if we can access a large spectral range where these objects emit (near infrared), one can get $T_{\text {ef }}(M)$ (flux variations as a function of $\lambda$ ) and the radius of the object (absolute value of emission knowing its distance from us).

An accurate knowledge of the object mass is fundamental to get a coherent model of internal structure and atmosphere. The observation of gravitationally linked systems (multiple systems) is thus a good way to get the mass, without the ambiguity of $\sin (i)$ in the radial velocity method.

The study of the composition and structure of the atmosphere is important for two main reasons:

- The atmosphere controls the loss of energy (coming from gravitational effects and nuclear reactions). The value of $T_{\text {ef }}(M)$ depends strongly on atmospheres.

- Clouds are forming in the atmosphere and modify the structure of molecular features (for instance $\mathrm{CH}_{4}$ ). However, the molecular features analysis is the basis of atmospheric modeling. From an observation point of view, one particular mean to study atmospheres is spectroscopy that allows determining the presence of several molecular compounds. Spectroscopic observations and modeling confrontation allow understanding the internal structure of the objects.

Clouds in the atmosphere of brown dwarfs The question of clouds formation in the atmosphere of brown dwarfs is particularly important because it mainly determines their emission spectrum in the infrared and the level of parent star reflected light in the visible spectral range. An important cloud cover leads to a flat spectrum.

Clouds form by the condensation of refractive compounds (silicates, $\mathrm{Al}_{2} \mathrm{O}_{3}$, other oxides, metals...) Grains tend to deposit thanks to gravity and mixed (back to upper atmosphere) thanks to turbulence. Depending of the description of these phenomena, atmospheric models lead to different spectral distribution in the thermal infrared (Fig. 4).

The measurement of infrared emission spectrum of low mass stars and brown dwarfs for which mass are well known would strongly help constraining models. It is important to cover a large sample of mass and thus a large sample of $T_{\text {ef }}$.

$\mathrm{CH}_{4}$ in the atmospheres Thermodynamics tells us that $\mathrm{CH}_{4}$ formation happens at a pressure where the gas temperature cools down to $1,400 \mathrm{~K}$. However, the relation between $T_{\mathrm{ef}}(M)$ and the temperature of the atmospheric parts responsible for infrared emission depends on atmospheric structure and particularly clouds location.

Comparing the presence of $\mathrm{CH}_{4}$ features in both observed and computed spectra allows determining an accurate structure of the atmosphere.

The observation of the methane $3.5 \mu \mathrm{m}$ spectral band (fundamental stretching mode of $\mathrm{C}-\mathrm{H}$ ) is particularly important because it is the spectral feature with the stronger oscillator strength. It is thus an accurate tracer of $\mathrm{CH}_{4}$ 
Fig. 4 Energy spectral distribution at $T_{\text {ef }}=2,000$, 1,500 and $1,100 \mathrm{~K}$ for different models: condensation and sedimentation (light blue), condensation and fixed aerosol (red), and sedimentation and turbulence (model "AMES settl"—dark blue). From [2])

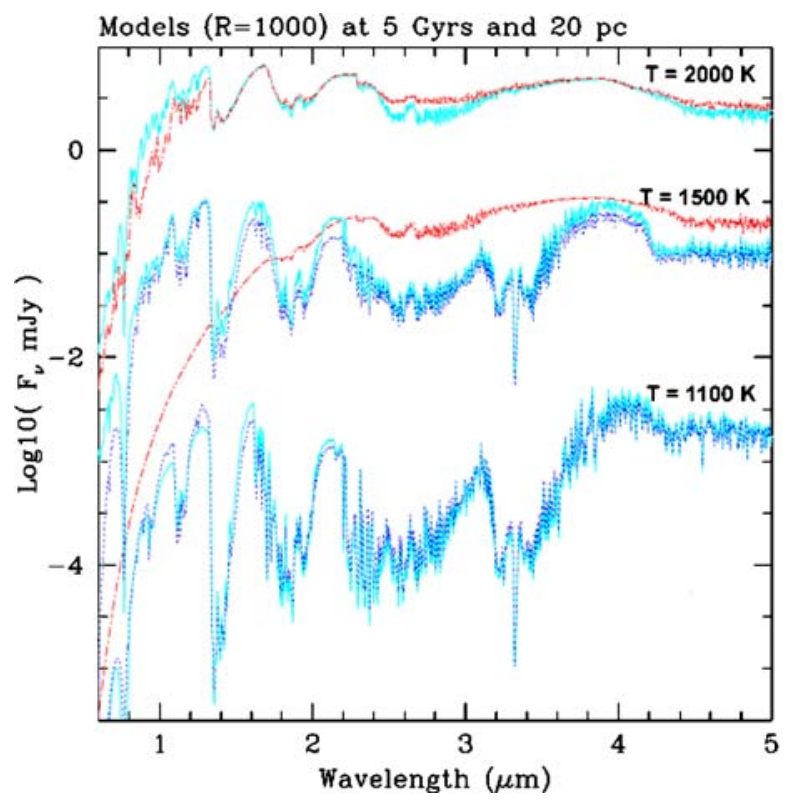

presence. This features cannot be observed from ground and require a space observatory.

Young linked objects If brown dwarfs are observed in star forming regions, the temporal dependence of the models can be studied (Fig. 5). This variable is fundamental for evolution models.

Fig. 5 Contrast brown dwarf/sun in the $L^{\prime}$ spectral band $(3.8 \mu \mathrm{m})$ as a function of mass and age of objects (from Burrows et al. models)

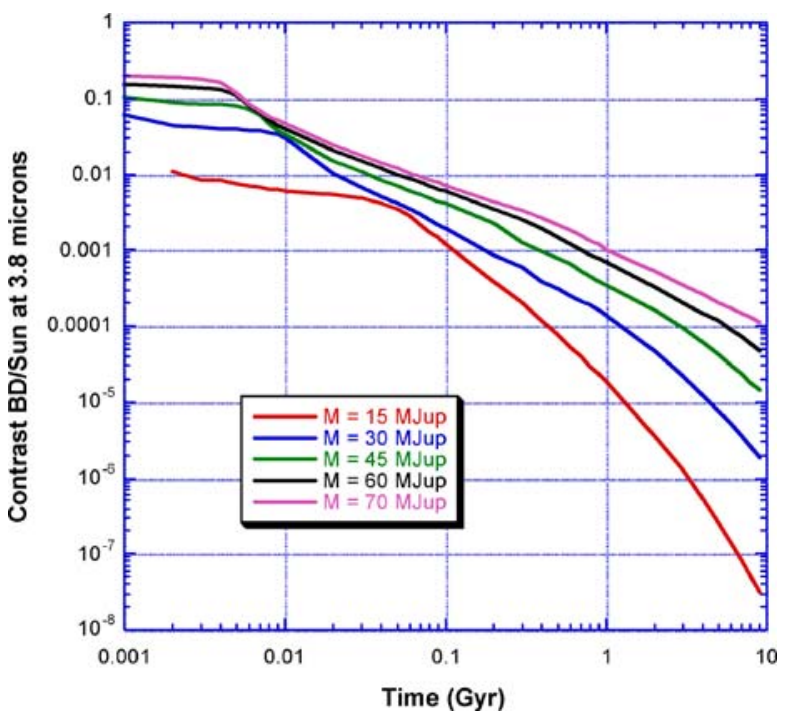


It is thus useful to observe in star forming regions (e.g. Taurus cloud) where systems with an age between 10 and 100 Myrs can be observed. The age of the object can be determined by the spectral analysis of the parent star, which is more massive and thus already in the main sequence in stellar evolution.

The study of $T_{\text {ef }}(M, t)$ and $R(M, t)$ and spectra as a function of the mass and age is possible in the star forming regions if the required angular resolution can be achieved. This requires interferometric observations, with baselines reaching several hundreds of meters. At present, no such objects are known in star forming regions, but it is probable that radial velocity survey identify a few of them in a near future. In that case, their spectroscopic study would be a real breakthrough.

\subsection{Other programs}

Taking into account the specificity of PEGASE instrumental concept and the high angular resolution and dynamics capabilities, other astrophysical goals can be considered:

- Active galactic nuclei: it is not possible to look at the accretion disk around the super massive black hole, but it is possible to study the dust dense structure around the central engine usually considered as an opaque torus.

- Resolving images of quasars split by gravitational lens effects. A cloud of intergalactic hydrogen of mass $M$ at half the distance from a $z$ red shifted quasar creates a gravitational splitting of its image with an angular separation equal to $\left(z \times M / 10^{4} M_{\text {sun }}\right)^{1 / 2}$, i.e. typically 1 to 3 mas for $M=10^{4}$ to $10^{5} M_{\text {sun }}$ and $z=1$ [9]. Observation can be considered with PEGASE, for brightest objects (magnitude equals 13.5 in the $\mathrm{K}$ band), assuming integration time of several hours and visibility accuracy of $1 \%$ (see Fig. 10). The angular resolution provided by PEGASE, up to a fraction of mas at a wavelength of $1.5 \mu \mathrm{m}$, is more five times better than VLBI at wavelength of $21 \mathrm{~cm}$.

- Temporal characterization of different classes of rapid pulsators: Rapid radial pulsators (from a few hours to a few days) with a limited angular diameter (from a few mas to a few hundredth of mas) appear naturally as excellent targets for PEGASE in this mode. The large spectral coverage from visible to mid-IR will ensure a simultaneous observation, which will allow, at a first level, to characterize the radial symmetric intensity distribution and/or the dependence of the source appearance with the wavelength.

- Disc formation and evolution of active hot stars.

\section{PEGASE: mission concept}

The mission profile and concept were studied during a CNES phase 0 in 2005 [13]. 


\subsection{Payload}

The PEGASE payload can be seen has a combination of three instruments:

- An IR interferometer (here after referred to as IR or main instrument),

- A cophasing interferometer (hereafter referred to as FS for fringe sensor),

- A fine pointing camera (hereafter referred to as FRAS for Fine Relative Angle Sensor).

The IR instrument performs precise measurements either in a stellar interferometry like mode (square visibility measurement) or destructive mode (nulling) in the [1.5-6 $\mu \mathrm{m}]$ spectral range. Using the [0.8-1.5 $\mu \mathrm{m}]$ range, the FS is used to precisely cophase the IR instrument but it also has scientific by-products. The FRAS operates in the $[0.6-0.8 \mu \mathrm{m}]$ range and is used to precisely correct the tip/tilt errors in order to ensure a very good intensity matching between the two arms. Both FS and FRAS have associated fine control mechanisms located in the payload.

The control aspects are so intricately linked with the satellite level that they will be presented later in this text although the fine control loops are located in the payload itself.

\subsubsection{Instrument concept}

The IR interferometer is a classical Michelson based on the principle illustrated by Fig. 6 . Two apertures separated by the baseline vector $B$ collect the coherent photons from a narrow field of view centered on a bright source and send them in a combining set-up where optical delay lines can precisely adjust the geometric optical path difference (opd). The detected signal is the intensity of the interferogram as a function of the opd, measured in various spectral channels.

Fig. 6 The basic instrumental principle

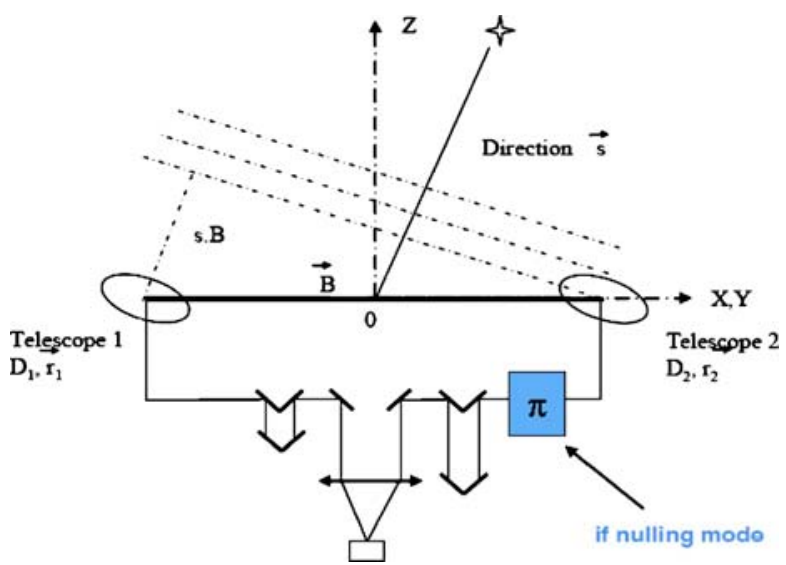


The visibility measures the contrast of the fringes and is defined in a simple way by:

$$
V=\frac{I_{\max }-I_{\min }}{I_{\max }+I_{\min }}
$$

A more precise definition generalizes this quantity and relates it to the Fourier transform of the source brightness. Assuming a rectangular bandpass, the equation of the fringes generally has the following form:

$$
\begin{gathered}
I(\delta)=I_{0}\left[1 \pm V_{\mathrm{obj}} V_{\varphi} V_{\mathrm{jit}} V_{\rho} V_{\mathrm{pol}} \sin c\left(\frac{\delta}{\wedge}\right) \cos \left(\frac{2 \pi \delta}{\lambda}\right)\right] \\
\text { with } \sin c(x)=\frac{\sin (\pi x)}{\pi x}
\end{gathered}
$$

$I_{o}$ is the mean collected intensity, which depends on the central target magnitude in the considered spectral range, the size of the collectors, the optical throughput and the quantum efficiency. $\delta$ is the optical path difference between the two wavefronts. It is adjusted by the delay lines. The contrast of the fringe is affected by various factors:

- $V_{\mathrm{obj}}$ is the visibility of the central object. This quantity is unity for a point source and has characteristic features for various shapes of the observed object. Measuring the quantity for various baseline vectors allow a reconstruction of the source image and an estimation of its physical parameters by fitting various theoretical models. The instrumental errors also impact the contrast: wavefront errors $\left(V_{\phi}\right)$, opd jitter $\left(V_{\mathrm{jit}}\right)$, intensity mismatch $\left(V_{\rho}\right)$ and differential polarization effects $\left(V_{\text {pol }}\right)$.

- In a polychromatic case, the fringes have a finite length support characterized by the coherence length: $\wedge=\lambda_{0}^{2} / \Delta \lambda=\lambda_{0} R$.

The PEGASE interferometer can be used in two ways:

- In the $\mathrm{V}^{2}$ mode, the instrument is used to extract $V_{\text {obj }}$ from a measurement of four points (ABCD) of the central fringe [19]. The instrumental errors limit the precision on the absolute measurements but because of its stability, it can be calibrated at the beginning of the instrument life. The measurement of the object phase as a function of the wavelength is not studied in our baseline proposal, but it can be considered as another method of detection (differential phase interferometry) after proper calibration.

- In the nulling mode, an achromatic $\pi$-phase shift is introduced to centre the dark central fringe on the bright central objects. The size and orientation of the baseline is adjusted to place the first bright fringe on the faint companion. If the instrumental errors are sufficiently small to ensure a stable fringe contrast greater than the contrast between the star and its weak companion, the spectrum of the faint companion can be extracted with a modulation of the interferometer. This modulation can be implemented by a slow rotation of the interferometer around the line of sight [7], Fig. 7). 


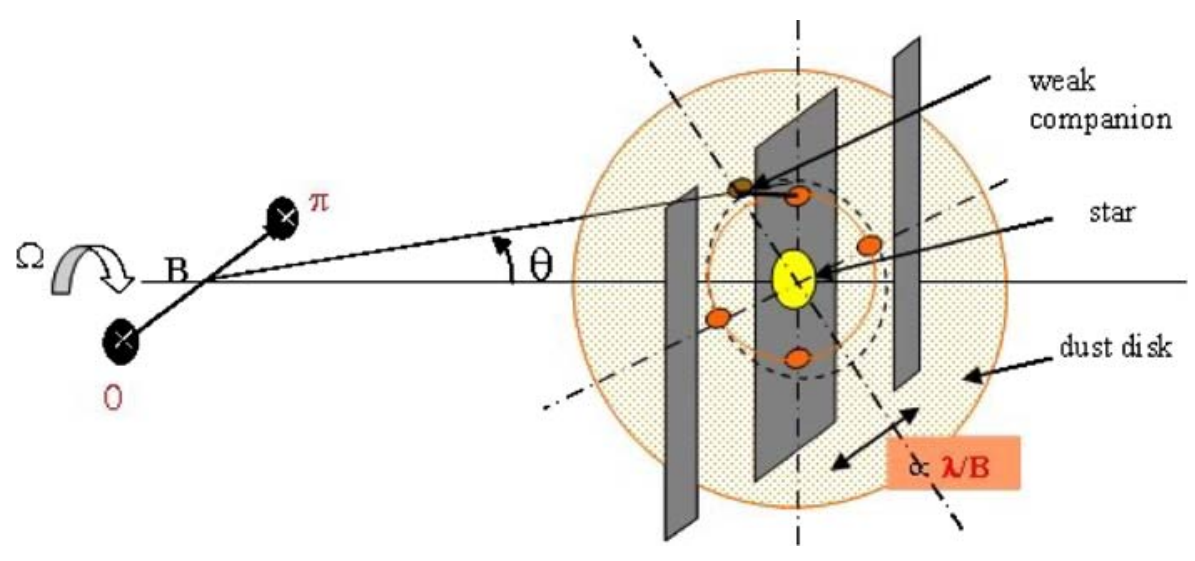

Fig. 7 The Bracewell concept

As the nulling mode of PEGASE focuses on relatively bright $\left(10^{-4}\right.$ to $10^{-3}$ contrast) and isolated faint companions in regions (stellar neighborhood) where the exo-zodiacal light is not disturbing, a simplified implementation is a limitation of the modulation to a finite number of baseline orientations. The minimum is two: one for noise calibration with zero transmission on the companions ( $B$ is orthogonal to the star-companion direction), and one for the measurements of the companion's signal. Some additional points may be added to improve the calibration and to tune the size of the baseline to the observed scene.

In the case of Darwin, the Bracewell principle fails to characterize the exoearths mainly because of the predominance of the exo-zodiacal light that is much more important than in the PEGASE case [15]. Nevertheless it is interesting to note that the most recent studies of Darwin converge toward a kind of double Bracewell set-up with four collectors coupled by pair. The outputs of the two Bracewell are coherently superimposed and modulated to overcome the exo-zodiacal light perturbation. From a technological and instrumental point of view, PEGASE in the nulling mode is then a very meaningful in flight demonstrator, but in a less technologically constraining spectral range.

\subsubsection{The IR interferometer}

Key characteristics The PEGASE IR interferometer in its linear configuration will use baselines varying from $B=40$ to $500 \mathrm{~m}$ and operate in the $1.5-6 \mu \mathrm{m}$ spectral range with a spectral resolution $R=60$. It is conceivable to consider a minimal baseline reduced to $20 \mathrm{~m}$ if a triangular configuration is adopted, with the beam combiner at one angle. In that case, the payload has to be designed with an angle of $120^{\circ}$ between the incoming beams instead of the $180^{\circ}$ in the linear case. This point has still to be assessed but would strongly 
increase the performance required for the exozodiacal cloud observation program. The spatial resolution can be adapted to the observed scene and may vary between 0.5 mas $(1.5 \mu \mathrm{m}, B=500)$ to 30 mas $(6 \mu \mathrm{m}, B=40 \mathrm{~m})$. The resolution can be lowered down to 150 mas in the nulling mode but with some SNR loss, in a 1 arcsec field of view (Airy disk size for individual telescope) by the use of next order dark fringes of the transmission map

The collecting diameter is limited to $D=40 \mathrm{~cm}$ due to the accommodation of the three spacecraft under the Soyuz fairing without deployable systems. Allowing a deployment of the siderostat mirrors from horizontal to $45^{\circ}$ angle is a first step that could increase $D$. A greater diameter would imply deployable sunshields and the limit would be fixed by the total launch mass. Only more detailed mechanical studies can fix the exact limit and $40 \mathrm{~cm}$ is proposed in a conservative way. The performance assessment is based on this value.

The cophasing (control of the $V_{\text {jit }}$ term of (2)) is performed by an internal opd fine control loop based on a fringe sensor measuring the observed central target in the NIR range and an optical delay line (ODL). The intensity control is performed by a fine pointing loop using the FRAS operating in the visible range and fast steering mirrors based on piezoelectric devices (FSM).

The proposed combination system is a pupil plane set-up based on a Modified Mach Zehnder device (MMZ) with a maximal integration of the IR and FS channels to guarantee a high opd stability between them. A Modal filtering is used to relax the optical WaveFront Error (WFE) constraints applying on the optics. The wavefront errors $\left(V_{\phi}\right.$ term of (2)) translate into intensity mismatch errors, which are easier to manage. The IR detection is based on an $\mathrm{HgCdTe}$ array detector of the Hawaii type with a low-resolution spectrometer based on a prism.

An overview of the optical bench A preliminary optical layout is illustrated by Fig. 8. The concept is driven by compactness, symmetry of the reflection in the two arms, stability of the opd, minimization of the coupling between the opd and tip/tilt correction.

Siderostat: $\mathrm{M} 1$ is a flat mirror with $45^{\circ}$ angle. Its size $(70 \times 50 \mathrm{~cm})$ is imposed by the accuracy of the relative spacecraft positioning.

Afocal: M2 and M3 form a simple afocal. The optical magnification $M$ will be a trade-off between dynamics of the tip-tilt errors, the available stroke of the fast steering mirrors, the actuation noise, the mechanical constraints and the polarization limitations. $M=20$ is today considered as a good starting value.

Achromatic phase shifter (APS): M4 is a small flat mirror, which directs the rays towards the active stage. Combined with the M1 layout, it creates an achromatic $\pi$ phase shifter using field reversal by reflections [6], one may not exclude the need to compensate small chromatic effects by dispersive prisms like the ones used by the MAII breadboard [23].

Active Mirrors (FSM): The FSM are to be placed the closest to the afocals in order to minimize the optical space were the tip/tilt errors are not corrected, and hence reduce differential polarization effects. Possible locations are M3 


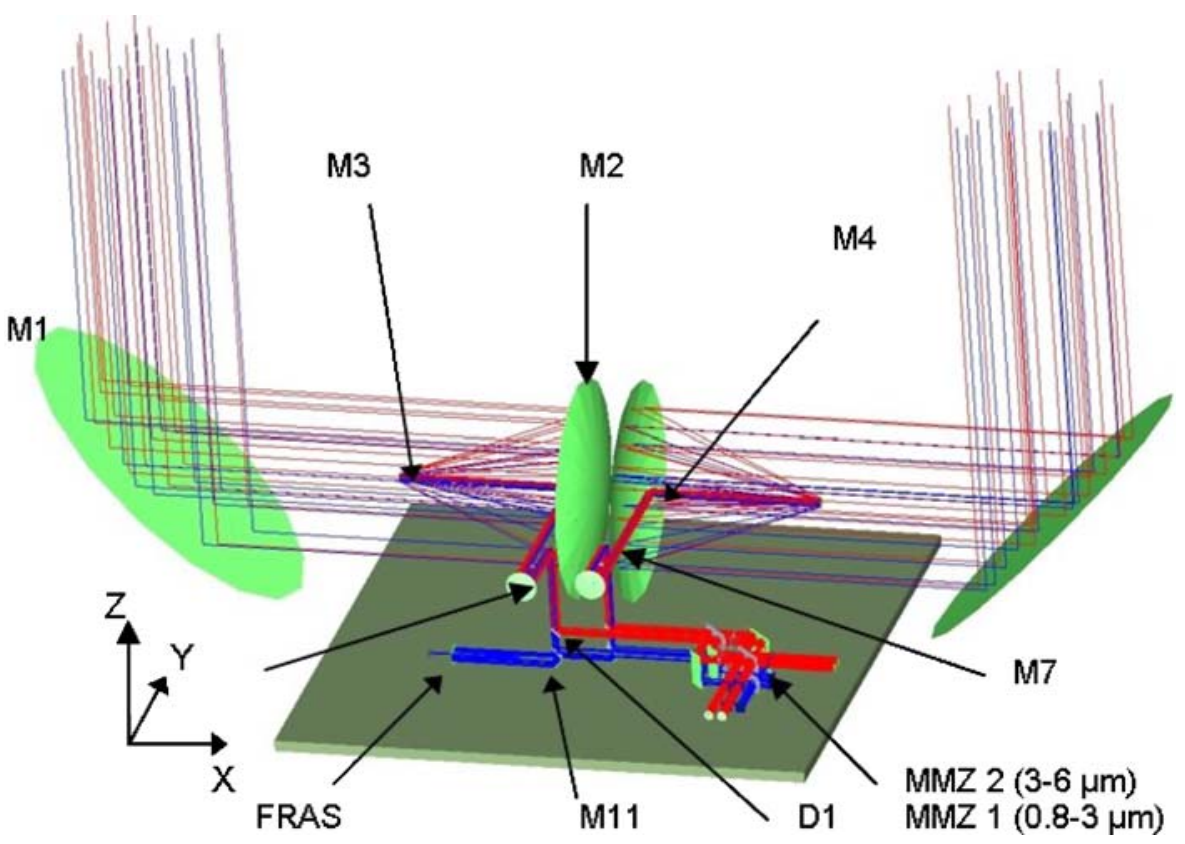

Fig. 8 Preliminary optical layout (see the text for a detailed description)

or M4. The optical stop is imposed on the FSM in order to reduce the coupling between tip/tilt and opd corrections.

Optical delay lines (ODL): M5 and M6 form a cat's eye type ODL. The optical combination has low incidence angles, which help to reduce the differential polarization effects. They are placed after the FSM to operate in a tip/tilt corrected optical space.

Combining stage (MMZ): A dichroïc plate reflects the wavelengths below $0.8 \mu \mathrm{m}$ to the FRAS and transmits the higher wavelengths toward the MMZ. Depending on the coating feasibility, one or two MMZ will be needed to cover the full wavelength range.

Injection stage: Small off axis parabolas focuses the four outputs of the MMZ into single mode fibers. A fluoride glass fiber can cover the spectral range 1 (1.5-3 $\mu \mathrm{m}$ range). A chalcogenide fiber is required for the spectral range 2 (3$6 \mu \mathrm{m})$.

Detection module: The fibers are connected to a detection stage including a dispersing prism and an $\mathrm{HgCdTe}$ array detector. For each spectral range, four columns correspond to the four outputs of the MMZ. Each column has 40 pixels corresponding to $R=60$. To cope with SNR requirements, the temperature has to be controlled at $T=55 \pm 0.1 \mathrm{~K}$.

This module is not represented on Fig. 8 but will lie toward the $-Y$ side of the bench, with a high thermal decoupling and a link to a sufficient radiator on the $+Z$ face. 
Internal loops sensors: The visible/NIR part of the spectrum is divided between the FS and the FRAS. These equipments are described in Section 3.1.5.

Structure: the payload requires a very stable optical bench made of Cesic. Our analysis shows that a $1.2 \times 1.2 \times 0.6 \mathrm{~m}$ size is compatible with the accommodation of the payload equipments if $D=40 \mathrm{~cm}$. To cope with the SNR requirement, the temperature of the optical bench has to be controlled at $T=90 \pm 1 \mathrm{~K}$. But as at $90 \mathrm{~K}$ the Cesic CTE is $3 \times 10^{-8} / \mathrm{m} / \mathrm{K}$, the temperature between the two arms have to be symmetric within a $0.1 \mathrm{~K}$ difference to match the opd stabilization specification.

Electronics: To avoid dissipation sources and a low working temperature, electronics have to be located outside the optical bench, in an electronic bay located in the service module.

\section{Operation modes}

Visibility mode: In the Visibility mode, the interferometer measures the four ABCD points of the central fringe in various spectral channels and for various baselines. Each baseline corresponds to a $1 \mathrm{~h}(1 \%$ precision $)$ to $10 \mathrm{~h}(0.1 \%$ precision) exposure. Ten baselines configuration (five sizes, two orientations) are planned to explore the spatial frequency domain. Exposure time is reached by adding individual $100 \mathrm{~s}$ integrations, the interferometer being re-co-phased between each elementary integration.

Nulling mode: The baseline size is optimized to adjust the first bright fringe projected on the sky at $3 \mu \mathrm{m}$ to the elongation of the weak companions. The first exposure uses a baseline orientation with a zero output for the companion and is used to calibrate the mean overall noise. The second exposure uses an orthogonal orientation to collect photons from the weak companion. A third exposure may be necessary to refine the optimal baseline. Each exposure lasts $10 \mathrm{~h}$. This optimization of observation, required for spectroscopy is performed after identification of the target parameters using ephemerides.

Performance assessment with respect to science objectives Various detailed SNR calculations were performed by different teams either in the laboratories or at CNES for a set of identified targets (hot jupiters, brown dwarfs, disks around T-Tauri star) (see by instance Le Duigou et al. [13]. Opd and tip/tilt performance are discussed later.

Nulling mode: The goal is to reach SNR $=10$. Towards the lower wavelengths, the performance is limited by the stability of the opd. For the highest wavelengths, the temperature of the optics and its stability limit the performance. In the central part, it is limited by the temperature of the IR detector and its stability but only for targets further than $50 \mathrm{pc}$. The performance can be described in a contrast (nulling difficulty) $J$ magnitude domain (cophasing difficulty) as illustrated in Fig. 9 (10 h exposure). Three categories can be defined: easy target ( $A$, blue), middle class target ( $B$, green), and difficult 
Fig. 9 Performance in nulling mode $(\mathrm{Ti}=10 \mathrm{~h})$. See the text for class description

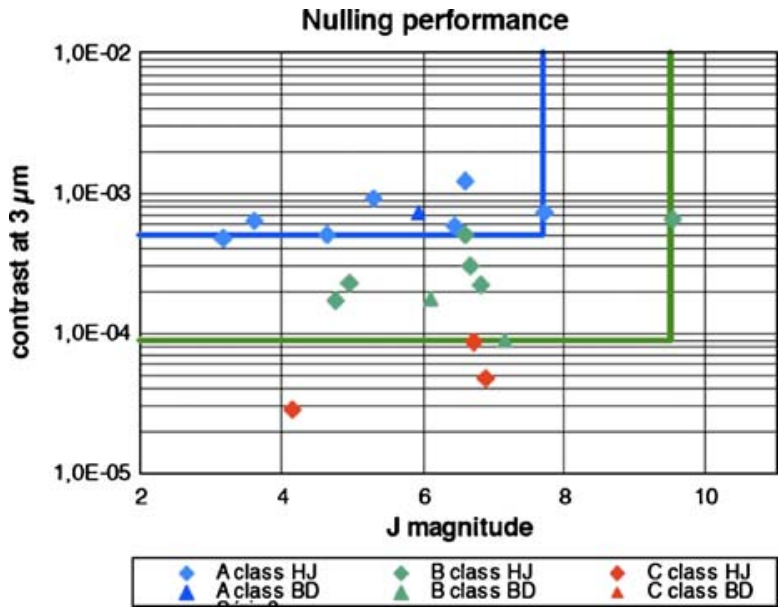

target ( $C$, red). They correspond respectively to a mean $1.5-6 \mu \mathrm{m}$ SNR greater than 10 , between 5 and 10 and below 5 . The $1.5-2.5 \mu \mathrm{m}$ SNR is lower and only class $A$ targets are accessible but with a degraded precision (SNR from 3 to 6 ). In 2005, 12 hot jupiters and three brown dwarfs were found in the $A$ or $B$ class with ecliptic latitude $\beta<30^{\circ}$. About twice that amount is now expected from recent extension of the target list.

Visibility mode: As the V2 mode has a bright fringe centered on the central object, the noise is dominated in most cases by the shot noise. The performance can be illustrated in an integration time/ $K$ magnitude domain (Fig. 10). The curves correspond to a given desired precision on the relative visibility measurement assuming a given mean visibility in $1.5-6 \mu \mathrm{m} .1 \%$ precision measurements are achievable within a $1 \mathrm{~h}$ integration time up to $K=8$ on extended objects with a mean visibility greater than 0.2 . This corresponds by

Fig. 10 Integration time in $\mathrm{V}^{2}$ mode as a function of $\mathrm{K}$

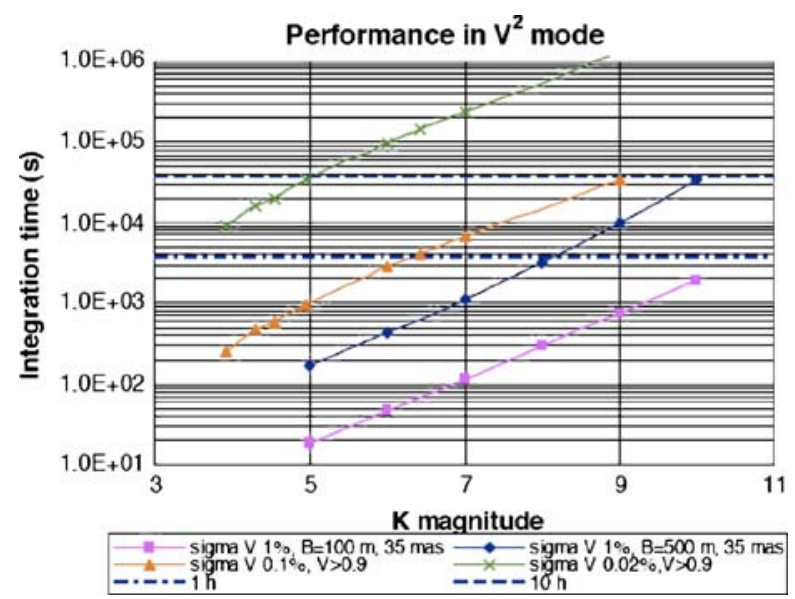


instance to a proto-planetary disk in the Taurus star formation region with $B=$ $500 \mathrm{~m}$ or to debris disks at $10 \mathrm{pc}$ with $B=50 \mathrm{~m} ; 0.1 \%$ precision are obtained on stars up to $K=9$ within a $10 \mathrm{~h}$ exposure which allows the characterization of low mass companions using the V2 mode with less stringent requirements on the detector temperature but longer integration times. The relative visibility measurements turn to absolute measurements if the initial calibration of the instrument has been done and the required stability is reached.

\subsubsection{The fringe sensor}

The fringe sensor is the key equipment of the PEGASE payload. The measurement noise is the major contributor to the opd budget and is then a critical parameter. Furthermore the capacity of fringe acquisition with a high initial drift is also a crucial issue that simplifies the free flying control architecture at satellite level. Last, the long-term stability between the opd measured by the FS and the IR opd is a third major topic, as illustrated by the recent results of nulling breadboards [23].

Modes The FS shall provide the measurements of the opd control loop, which has two main modes:

- Fringe acquisition: the opd can be much larger than the coherence length of the FS. It is thus necessary to perform a fringe search, by moving the ODL or waiting for the fringes to pass while the spacecraft drift. Because of the size of the baseline, the speed $v$ of the fringes can reach several tens of micrometer per second.

- Fringe fine tracking: the goal is to stabilize the fringes at the $2.5 \mathrm{~nm}$ rms level. The stability can be split in two main terms: a noise (typical time scale is $100 \mathrm{~s}$ corresponding to elementary exposures) and a long-term drift (typical time scale of $10 \mathrm{~h}$ ).

Description and key characteristics In order to reach a high sampling rate with a minimum number of pixels, a coaxial beam combiner is the most relevant to combine the two beams. To cope with the stability requirements and the fast fringe drift, a spatial modulation is used without any moving part. Such a FS has already been investigated in our team for stellar interferometry on ground [8]. The FS uses two spectral channels with $R_{\mathrm{FS}}=2.5$. The fine tracking performance is better in the NIR because the central star is less resolved and the optical transmission is higher than in the visible. The light from each of the four $\lambda / 2$ phase-shifted output is dispersed and focused on four single-pixel InAsGa detectors per spectral channel.

The proposed recombination is an adaptation of the Modified Mach Zehnder set-up [18] made of $\mathrm{CaF} 2$ with an appropriate geometrical design to avoid straylight (see Fig. 11). It is a proposed improvement of the system, which was validated on the Synapse breadboard with a $3 \times 10^{-4}$ nulling in the $\mathrm{K}$ band [12]. In the IR band, it can provide two nulled outputs one of them 


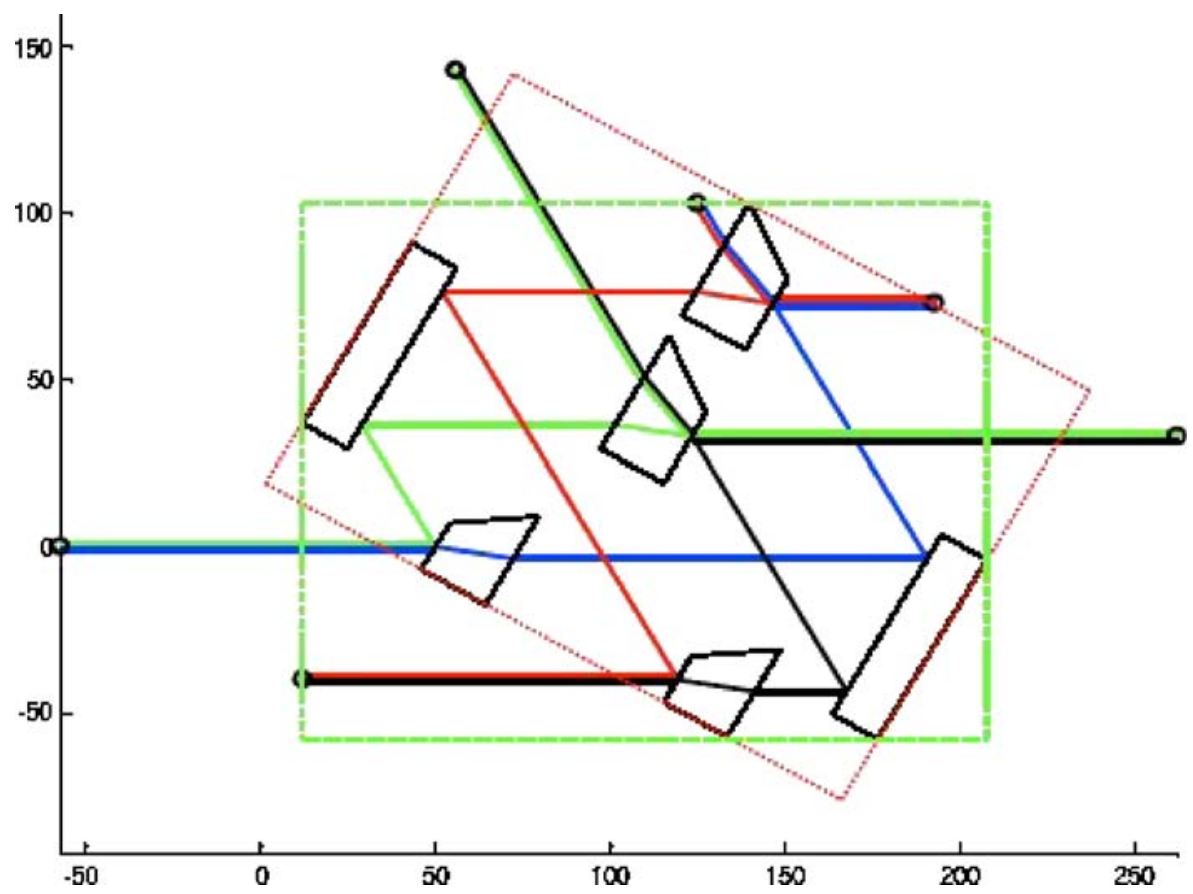

Fig. 11 Symmetrical MMZ recombination stage. The input is located at the left bottom side of the scheme, and the outputs are at the top and at left side of the scheme. Each ray (first reflected/transmitted then transmitted/reflected) is show by a different color. The green rectangle gives an idea of the breadboard size. Axes numbers are in millimeters

being achromatic due to a symmetric layout of the beam splitter parts. An adequate and very precise positioning of the mirrors enables to generate the four $\mathrm{ABCD}$ outputs spatially in the FS spectral range. The induced phase do not affect the IR nulled outputs and is chromatic in the FS band, but without hampering the detection performance. Both the FS and the IR bands share the same optics, which insures a very intrinsically stable system. The tight integration of these two functions is the heart of the payload as it reduces the differential stability requirements between the cophasing metrology and the IR interferometer. The difficulty translates to the optical coatings that have to cover the 0.8-6 $\mu \mathrm{m}$ range. Recent work carried at the Fresnel Institute show that solutions exists with a reasonable number of layers (40) but impacts on nulling may be too difficult too handle. This will be tested in the PERSEE breadboard (see Section 3).

Performance assessment This section is a summary of a study performed by ONERA under CNES contract.

Fine tracking mode: The main drivers are the magnitude of the central object, its visibility in the $1-1.5 \mu \mathrm{m}$ band with a baseline adjusted to the observed 
target in the nulling band, the ReadOut Noise (RON) of the detector, the total transmission, including the quantum efficiency. Figure 12a shows the performance in fine tracking mode with a $10 \mathrm{~Hz}$ sampling frequency and a RON of $20 \mathrm{e}^{-}$rms as a function of the $J$ magnitude. The calculations were made in the case of a central star with a visibility $V>0.8$ in the SF band. The noise is inversely proportional to $V$ in the case of more extended sources. An approximated law is given by (3):

$$
\sigma_{\text {opd }}=\frac{\left(0.02 J^{25}+0.05\right)}{V} \sqrt{\frac{f_{\mathrm{s}}}{10}}
$$

The $2.5 \mathrm{~nm}$ rms level corresponds to $J=7$ with $f_{s}=10 \mathrm{~Hz}$, where $f_{s}$ is the sampling frequency

Acquisition mode: To avoid blurring, the exposure time must be close to $1 \mathrm{~ms}$, and the repetition time small enough so that measurements correctly

Fig. 12 a and $\mathbf{b}$ Measurement noise in fine tracking mode at $f=10 \mathrm{~Hz}$ and with $\mathrm{RON}=$ $20 \mathrm{e}^{-} \mathrm{rms}$ as a function of $J$ (top). SNR in acquisition mode for various drift speeds and a sample of three stars with $J=4.6,6.9$ and 9.5 (bottom)
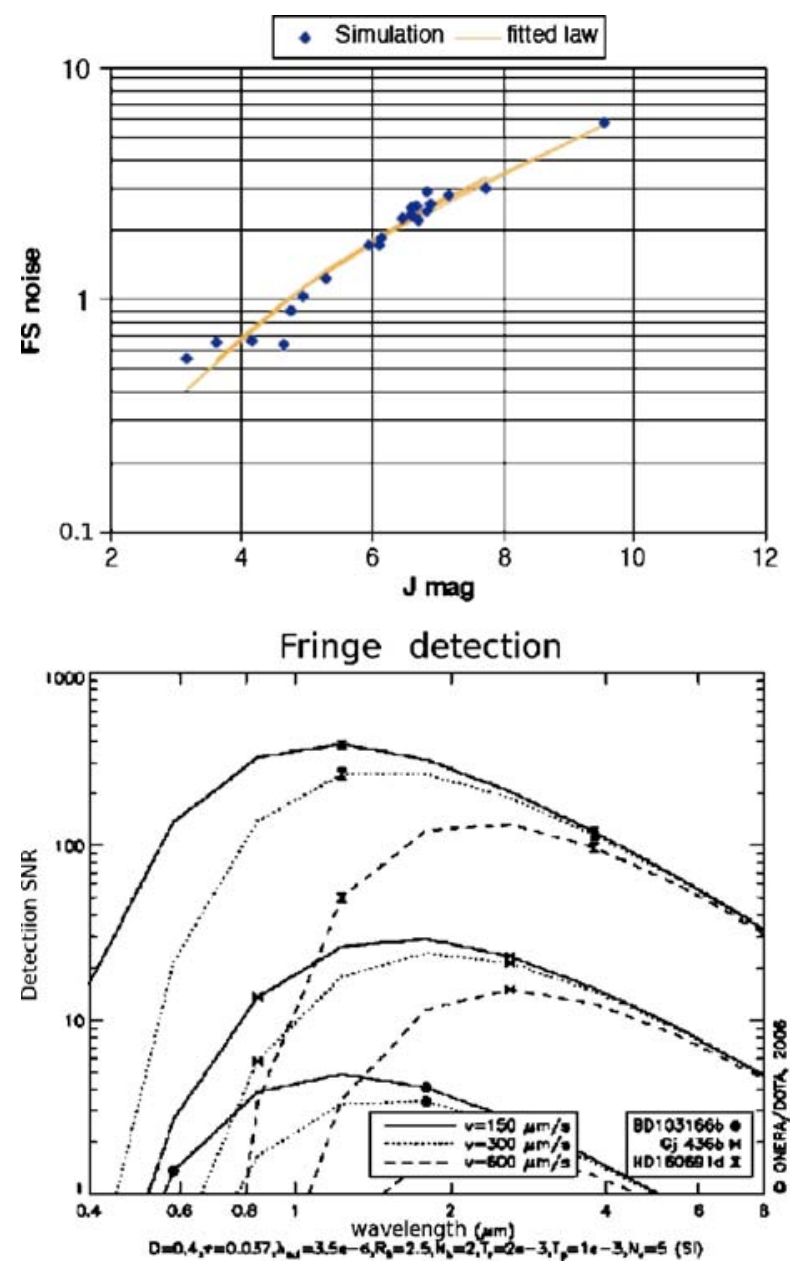
sample the chromatic envelope of the fringe pattern. Figure $12 \mathrm{~b}$ shows that for detection, the best spectral domain is shifted towards the IR, because the long wavelengths less suffer from blurring. Assuming a minimum SNR of 10 to detect the fringe, an OPD drift up to $150 \mu \mathrm{m} / \mathrm{s}$ is acceptable if $J<8$ and $V>0.8$.

\subsubsection{Delay lines $(O D L)$}

The requirements are a $\pm 1 \mathrm{~cm}$ stroke and $1 \mathrm{~nm} \mathrm{rms}$ resolution at $100 \mathrm{~K}$, with a close loop control bandwidth of $100 \mathrm{~Hz}$ corresponding to expected formation flying main perturbations spectral band. According to recent development at TPD-TNO under ESA contract, this is feasible with magnetic bearings and voice coil actuation [21]. The fine part could be mitigated with the FSM that can also provide a fine opd tuning along with tip/tilt correction if the stroke is sufficient.

\subsubsection{The FRAS}

Description and key characteristics The proposed approach to measure the tip-tilt errors consists in using the visible $0.6-0.8 \mu \mathrm{m}$ domain. The estimation of the error signal will be made with images recorded by a camera common to the two arms and located in an image plane: the field relative angle sensor (FRAS). It can be based on a Cassegrain type small telescope with $f=0.5 \mathrm{~m}$ and a classical 1,024 $\times 1,024 \mathrm{Si}$ based CCD (47-20 from E2V) or CMOS (HAS from Fillfactory) where one pixel represents $0.3 \mathrm{arcsec}$ on the sky. The FRAS loop has two main modes: star acquisition and fine pointing.

Performance assessment in the fine pointing mode This section is a summary of a study performed by ONERA under CNES contract [8].

Specification: Starting with the residual rotations of the spacecraft, the fine pointing loop has to ensure a symmetrical coupling into the monomode fibers and a minimal loss of flux (transmission $>0.7$ from 1.5 to $5 \mu \mathrm{m}$ ). Assuming that the coupling efficiency follows the Strehl approximation and a mean intensity mismatch of $10^{-2}$, with a $3 \times 10^{-3}$ stability, a specification of around 30 mas rms per axis on the sky for the pointing stability has been evaluated for $M=20$. The residual bias after calibration shall be of the same order of magnitude.

Noise estimation: The photon noise is obtained from the $V$-magnitude of considered stars and an estimation of the total transmission from the stellar wavefront to the detector of $40 \%$. A classical value of $15 \mathrm{e}^{-}$rms is assumed for the RON. Under these assumptions, the noise of the FRAS is lower than 10 mas rms for a sampling frequency as high as $100 \mathrm{~Hz}$ for $V_{\text {mag }}$ up to 8 .

FSM The tip/tilt error will be controlled in a feedback loop with two fast FSM based on piezoelectric actuators. Each FSM uses three piezoelectric 
actuators. The required dynamical range is \pm 40 arcsec but the total stroke shall also take into account some alignment corrections. The total required range is $\pm 100 \operatorname{arcsec}$ at $100 \mathrm{~K}$. The required resolution is $0.3 \operatorname{arcsec},(1 \mu \mathrm{rad} \mathrm{rms})$. This specification is well within the capabilities of existing tip/tilt actuators (see by instance the PI S 316.10 with $0.2 \mathrm{~nm}$ rms resolution, $10 \mu \mathrm{m}$ linear stroke, \pm 250 arcsec angle stroke). Differential polarization calculations show that such angles are compatible with the nulling specification.

\subsection{Spacecraft key factors}

The PEGASE mission is composed of three satellites: one combiner and two siderostats, which are identical spacecraft but with symmetric payloads. As they fly in precise formation, some emphasis will be put on this aspect in the following sections.

\subsubsection{Attitude and orbit control required and associated requirements (free-flying)}

Architecture trade-off Two R\&D studies were dedicated to the Guiding and Navigation Control (GNC) of PEGASE and are summarized in [22]. Two main architectures are in competition to achieve the ultimate control goals:

- Continuous control, opd fine loop inside the payload,

- Dead band control and reaction wheels, opd and tip/tilt fine loop inside the payload.

The first one relies only on control at satellite level for pointing with a 10 mas pointing requirement, a $1 \mu \mathrm{N} / \mathrm{sqrt}$ (hertz) actuation noise and very precise laser longitudinal and lateral metrologies. This option is technologically very demanding. That is why we considered another solution with a mitigation of the pointing control between the spacecraft and the payload. It relaxes the metrology and actuation constraints at satellite's level and allows the use of more conventional technologies. As a pulsed control for all the 18 degrees of freedom (dof) is impossible due to synchronization problems, a continuous control based on reaction wheels on the siderostats is used to control their relative pointing. This option is presented here as our baseline proposal, although the full trade-off can only be closed by more detailed studies.

\subsubsection{Formation modes}

A proposed architecture of the main GNC modes is illustrated by Fig. 13 [22]. In the transfer/deployment mode each satellite movement is only governed by the gravity laws, its own propulsion and the ground segment commands. Each satellite has its own FDIR and link with the ground segment.

In the Formation Control Mode (FCM) first phase, the formation keeping relies on pulsed control using cold gas thrusters, star trackers and the radio frequency metrology that provides an accuracy of $1 \mathrm{~cm}$ in distance and of $\pm 1^{\circ}$ 
Fig. 13 GNC main modes for the three satellites (hub and siderostats) and their interactions (see the text for description)

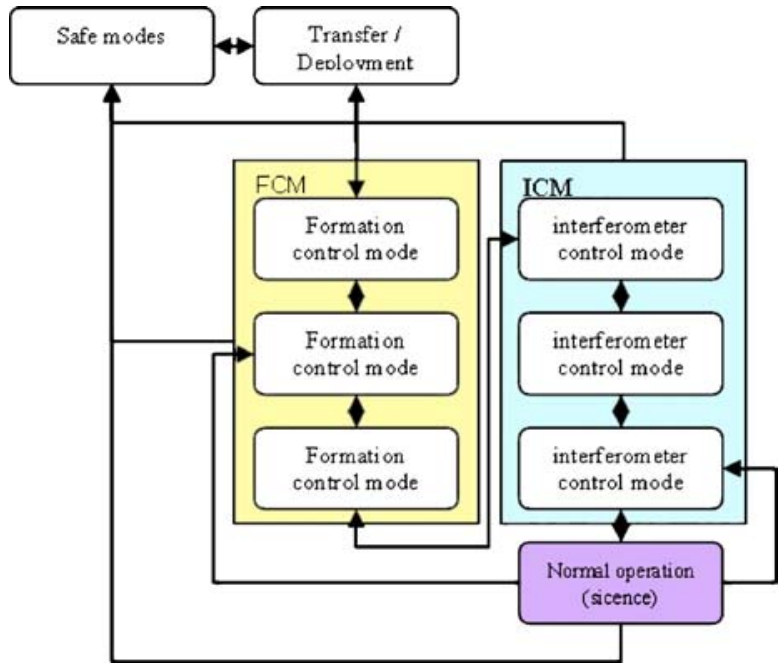

in lateral position. The pointing accuracy is about 15 arcsec for each spacecraft. In the FCM second phase, the optical sensor (Field Of View (FOV) of $3^{\circ}$ ) helps to reduce the lateral error down to the $10 \operatorname{arcsec}$ level $(1 \mathrm{~cm}$ at $250 \mathrm{~m})$. In phase 3 , the dead band control synchronization is performed.

The Instrument Control Mode (ICM) first phase consists in acquiring the guiding star in the FRAS and controlling the relative pointing of the siderostats continuously with reaction wheels. The information coming from the FRAS is then used in the payload to achieve the fine control with the fast steering mirror. In the ICM third phase, the opd drift is damped and the fringe sensor is used to achieve the fine opd control with the optical delay line.

In the nominal mode, the fine mechanisms have to compensate the external residual movements by corresponding displacements. They must have a high enough bandpass to reject noises with sufficient efficiency. As they have a finite stroke, they cannot compensate the spacecraft movements during an integration time of several hours. It has to be split into elementary integration periods during which the vehicles are left drifting under the natural perturbations. Off loading of the mechanisms is performed by adapting the thruster's pulses. They will create an OPD shift exceeding the $2.5 \mathrm{~nm}$ level and interrupt the scientific observation for a short time (loss of the fine fringe tracking but the OPD remains in the coherence length). The observation can be split in $100 \mathrm{~s}$ slices.

Filtering algorithms using the predictable movement in the L2 environment are used by the control to relax the resolution of the sensors imposed by the formation-damping requirement. Table 1

Performance estimation The performances of the proposed architecture were assessed in details by EADS-ASTRIUM [22]. The solar pressure noise is taken into account with a $3 \%$ discrepancy between the ballistic coefficients of the 
Table 1 Summary of the payload characteristics

\begin{tabular}{|c|c|c|c|}
\hline Parameter & Value & Parameter & Value \\
\hline Baseline size & $40-500 \mathrm{~m}$ & FRAS detector & $1,024^{\wedge 2} \mathrm{CCD}$ \\
\hline Optical bench mass & $70 \mathrm{~kg}$ & FRAS pixel scale, FOV & $0.3 \mathrm{arcsec} / 5 \mathrm{arcmin}$ \\
\hline Electronic bay mass & $25 \mathrm{~kg}$ & FRAS spectral range & $0.6-0.8 \mu \mathrm{m}$ \\
\hline Power budget & $100 \mathrm{~W}$ & FRAS perf. @ 10 Hz & $<10$ mas $\operatorname{rms}(V=8)$ \\
\hline Optical bench size & $120 \times 120 \times 60 \mathrm{~cm}$ & FS detectors & InAsGa \\
\hline Electronic bay size & TBD & FS spectral resolution & 2.5 \\
\hline Collector diameter & $40 \mathrm{~cm}$ & FS spectral range & $0.8-1.5 \mu \mathrm{m}$ \\
\hline Magnification of afocal & 20 & FS opd noise @ $10 \mathrm{~Hz}$ & $2.5 \mathrm{~nm} \mathrm{rms}(V=8)$ \\
\hline Effective focal length (IR) & $1,800 \mathrm{~m}$ & FS max. fringe drift & $150 \mu \mathrm{m} / \mathrm{s}$ \\
\hline Mirror coatings & Gold, $\lambda_{\text {vis }} / 20$ & IR detector & $\begin{array}{l}1,024 \times 1,024 \mathrm{HgCdTe} \\
\text { array }\end{array}$ \\
\hline Optics temperature & $90 \pm 0.1 \mathrm{~K}$ & IR spectral resolution & $60,1.5-6 \mu \mathrm{m}$ \\
\hline Detector temperature & $55 \pm 0.1 \mathrm{~K}$ & IR spatial resolution & $0.5-30$ mas \\
\hline IR total transmission ${ }^{\mathrm{a}}$ & $7 \%$ & Max Integ. time & $10 \mathrm{~h}\left(\right.$ nul. or $\left.0.1 \% \mathrm{~V}^{2}\right)$ \\
\hline FS total transmission ${ }^{\mathrm{a}}$ & $4 \%$ & IR Min. contrast & $10^{-4}$ \\
\hline FRAS total transmission & $40 \%$ & Nulling $2.5-5 \mu \mathrm{m}$ SNR & $>10$ \\
\hline Alignment precision & 5 arcsec rms by pairs & $\mathrm{V}^{2}$ measurement SNR & $>100(1 \%$ acc $)$ \\
\hline RON (IR, FS, FRAS) & $<15 \mathrm{e}^{-} \mathrm{rms}$ & $\mathrm{V}^{2}$ measurement SNR & $>1000(0.1 \%$ acc $)$ \\
\hline
\end{tabular}

Main characteristics of the payload

ancludes beam splitters and 0.7 fiber coupling, assumes 0.97 minimum reflectivity, include the quantum

spacecraft (mass, apparent surface, inertia coefficients...). The selected wheels are the TELDIX RSI-5/28 (0.12 N ms) capable of correcting pointing errors within bandwidth of $1 \mathrm{~Hz}$ maximum. The torque noise was modeled with a high accuracy. The micro vibrations were measured on ground and applied on typical transfer function with sun-shield modes at $20 \mathrm{~Hz}$. The cut-off optimal frequencies are $0.02 \mathrm{~Hz}$ for the relative pointing of the siderostat, $0.15 \mathrm{~Hz}$ for the fine tip/tilt loop and $1.5 \mathrm{~Hz}$ for the fine opd loop. An open loop command is sent to the ODL to compensate the constant estimated drift from differential solar pressure effects. The obtained performances are summarized in Table 2 and illustrated by Fig. 14 for a case with a galactic angle $\beta=30^{\circ}$ and a $J=6$ central star. The opd noise is dominated by the FS filtered noise. The micro vibrations are $<1 \mathrm{~nm}$ rms.

Sensors and actuators requirements From the analysis performed by Astrium, the requirements of Tables 3 and 4 are derived.

Table 2 Simulated GNC performance

\begin{tabular}{ll}
\hline Parameter & Performance \\
\hline Combiner pointing (max on $100 \mathrm{~s})$ & 8 arcsec \\
Relative pointing & $2 \mathrm{arcsec}$ \\
Longitudinal position (max) & $1 \mathrm{~mm}+$ bias \\
Lateral position (max) & $8 \mathrm{arcsec}+$ uncalibrated bias \\
Opd (rms) & $3 \mathrm{~nm}$ \\
Tip/tilt (rms on the sky) & $20 \mathrm{mas}$ \\
\hline
\end{tabular}


Fig. 14 Dead-band control synchronization for the hub attitude (top), each color corresponds to each rotation angle. Impacts of control pulses on the OPD control error (bottom). Upper curve shows the spikes generated by control pulses (every $100 \mathrm{~s}$ ). The maximum transient error is below $1 \mu \mathrm{m}$. The middle curve is a temporal zoom on one transient. Lower curve is a zoom on the control error during the free flying phase
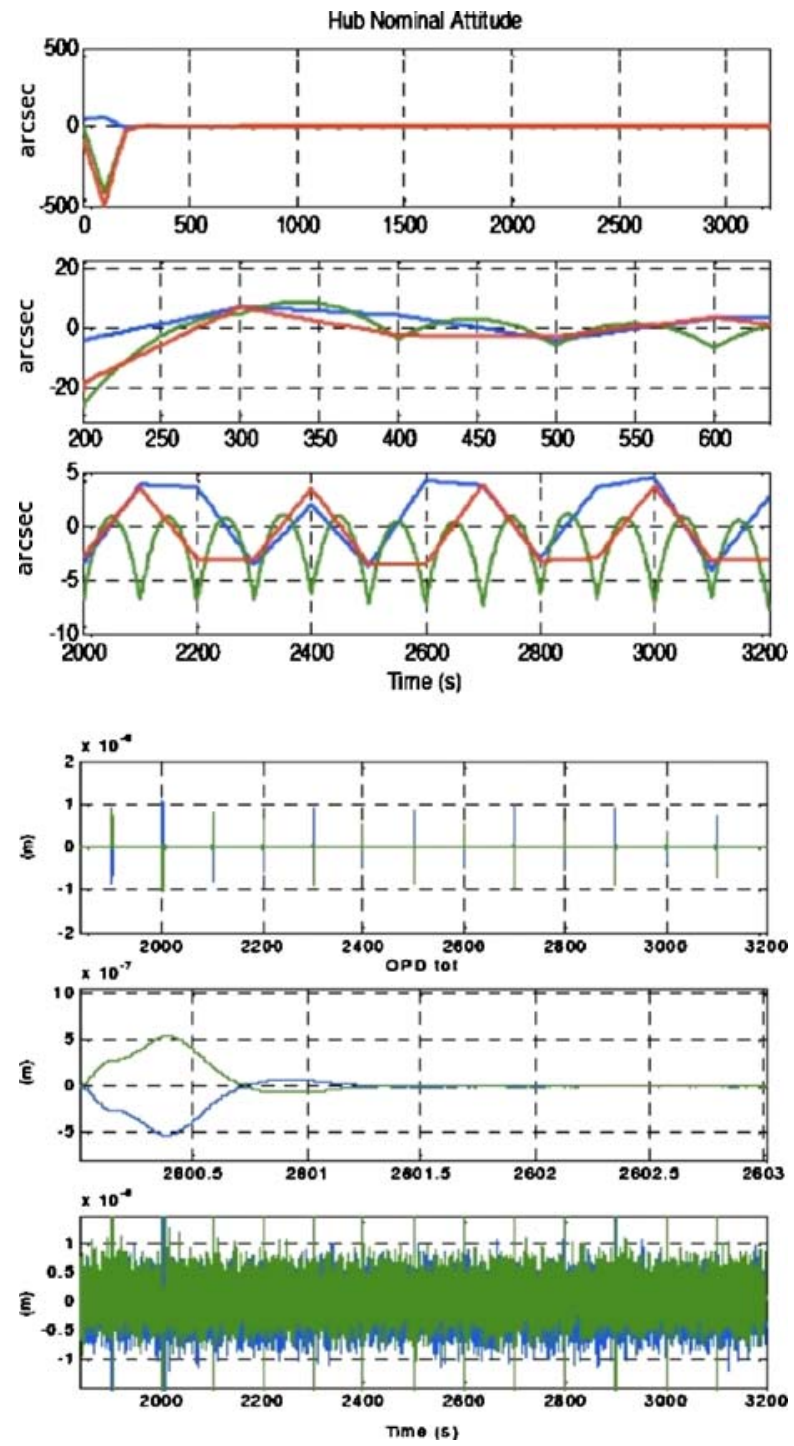

Fringe acquisition and associated calibration process The cumulated bias of the various metrologies induces an initial maximum bias of $\pm 8 \mathrm{~cm}$. As this exceeds the foreseen stroke of the ODL, a calibration process is needed. For the first fringe acquisition, and later at regular intervals during the mission, it is foreseen to perform a continuous scan the OPD using the movement of one siderostat in order to estimate this cumulated bias with a precision roughly equal to the coherence length of the fringe sensor (about $10 \mu \mathrm{m}$ ).

After this first calibration, before a routine fringe acquisition, the control residues insure a $\pm 3 \mathrm{~mm}$ range. The residuals of the calibration lead to a 
Table 3 Metrology requirements

\begin{tabular}{|c|c|c|c|c|}
\hline Metrology & Noise & $\begin{array}{l}\text { Bias after } \\
\text { calibration }\end{array}$ & $\begin{array}{l}\text { Sampling } \\
(\mathrm{Hz})\end{array}$ & Note \\
\hline $\begin{array}{l}\text { Siderostat star } \\
\text { tracker }\end{array}$ & 10 arsec/sqrt $(\mathrm{Hz})$ & $10 \operatorname{arcsec}$ & 10 & Standard SED 16 \\
\hline $\begin{array}{l}\text { Combiner star } \\
\text { tracker }\end{array}$ & $3 \operatorname{arsec} /$ sqrt $(\mathrm{Hz})^{\mathrm{a}}$ & $1 \operatorname{arcsec}^{b}$ & 10 & $\begin{array}{l}\text { Improved STR (Galileo } \\
\text { Avionics), located near } \\
\text { the payload for } \\
\text { stability reasons }\end{array}$ \\
\hline Optical metrology & $1 \mathrm{arsec} / \mathrm{sqrt}(\mathrm{Hz})$ & $1 \operatorname{arcsec}^{b}$ & 10 & Optical head on combiner \\
\hline Radio frequency & $3 \mathrm{~mm} / \mathrm{sqrt}(\mathrm{Hz})$ & $1 \mathrm{~cm}$ & 10 & \\
\hline FRAS & 10 mas/sqrt $(\mathrm{Hz})$ & 30 mas & 10 & Inside the combiner payload \\
\hline Fringe sensor & $0.56 \mathrm{~nm} / \mathrm{sqrt}(\mathrm{Hz})$ & - & 20 & Inside the combiner payload \\
\hline
\end{tabular}

${ }^{a}$ Initial spec. is 1 arcsec/sqrt (hertz) but sensitivity analysis show that the requirement can be somewhat relaxed

${ }^{\mathrm{b}}$ For a $1.25 \mathrm{~cm}$ lateral position specification as specified in the EADS study. This can be relaxed to at least $2.5 \mathrm{~cm}$ by increasing slightly the size of the siderostat mirrors by $2.5 \mathrm{~cm}$

$\pm 0.7 \mathrm{~mm}$ uncertainty. Thus, the required stroke of the ODL is $\pm 1 \mathrm{~cm}$. The initial opd drift is in the worst-case $30 \mu \mathrm{m} / \mathrm{s}$, which is well within the FS capability. A stepped scan is preferred to search for the fringes because it is simpler to implement and imposes less requirement on the FS.

The analysis shows that there is no need for a longitudinal laser metrology, either for bias reduction or damping requirements.

The bias of the star tracker can be calibrated using the method described in previous section. The bias of the optical sensor can be calibrated by moving one siderostat laterally and measuring the flux variation in the combiner.

\subsubsection{Propulsion}

We propose an impulsive cold gas $\left(\mathrm{N}_{2}\right)$ system based on existing technology (10 $\mathrm{mN}$ thrusters from Marotta UK) but with an improved Minimum Impulsion Bit (MIB) $(50 \mu \mathrm{N} \mathrm{s} \mathrm{m})$. Assuming $70 \%$ efficiency, the total $\Delta V$ needed for the formation control, the fine orbit control and the failure detection and recovery system is $25 \mathrm{~m} / \mathrm{s}$ for the combiner and $17 \mathrm{~m} / \mathrm{s}$ for each siderostat, according to our estimations. For high $\Delta V$ (injection, rough positioning) we propose a classical hydrazin system. The launcher corrections, the injection on the orbit and the orbital maneuvers require about $90 \mathrm{~m} / \mathrm{s} \Delta V$ on each spacecraft (assuming 98\% efficiency).

Table 4 actuators requirements

\begin{tabular}{|c|c|}
\hline Actuators & Performances \\
\hline Wheels & Torque noise $2 \mu \mathrm{Nm} / \mathrm{sqrt}(\mathrm{Hz})$ (allocation $0.77 \mathrm{~nm} \mathrm{rms}$ ), $\mu$ vibration $1 \mathrm{~nm} \mathrm{rms}$ \\
\hline Thrusters & Thrust alignment $2^{\circ}$, amplitude $1 \%, 5 \mu \mathrm{N}$, Mini Impuls Bit $50 \mu \mathrm{N}$ s \\
\hline ODL & Stability $1 \mathrm{~nm}$ rms, quantification $1 \mathrm{~nm} / \mathrm{s}$, dynamic stroke $\pm 1 \mathrm{~cm}$ \\
\hline Mirrors & Stability 10 mas rms, dynamic stroke $\pm 40 \operatorname{arcsec}$ \\
\hline
\end{tabular}




\subsubsection{Satellite overall architecture}

The satellites architecture is driven by the free flying requirements, the thermal stability, the accommodation under the Soyuz fairing with fixed sunshields. The thermo-mechanical architecture we propose is based on dedicated platforms with a maximum use of existing equipments. In the L2 case, the thermal control provides a high decoupling between the service module $(300 \mathrm{~K})$ and the optical payload (100 K) using V-grooves systems (Planck technology). The detector stage $(55 \mathrm{~K})$ is decoupled w.r.t. the optical bench and connected to a radiator. The $\mathrm{V}$-grooves and the payload have to be protected from the direct sun light by sun shields assuming anti-solar pointing with a maximum depointing angle of $30^{\circ}$. A preliminary architecture of the combiner is illustrated by Fig. 15.

\subsection{Orbit requirements}

During the Phase 0 at CNES various orbits were studied. The main drivers are the temperature stability of the payload and the gravity gradients required by the free flying. Two kinds of orbit are still in competition and a full trade-off would require more detailed studies, especially as far as thermal analysis is concerned.

\subsubsection{L2}

The very stable environment around the L2 point is of course the ideally suited place to perform an interferometric mission in the infrared. A 250,000 $\times$ $673,000 \mathrm{~km}$ Lissajou orbit was selected as the preferred option during the CNES phase 0 . It has an in-plane instable period of about 180 days and an

Fig. 15 Artist view of the combiner spacecraft

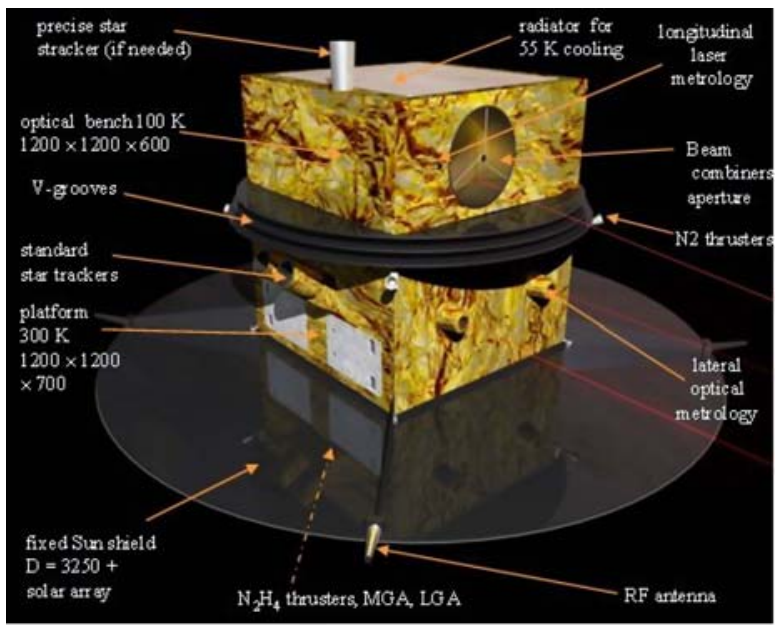

刑 Springer 
Fig. 16 a and b L2 Lissajou Orbit (top) and injection trajectory (bottom). The black circle is the orbit of the moon
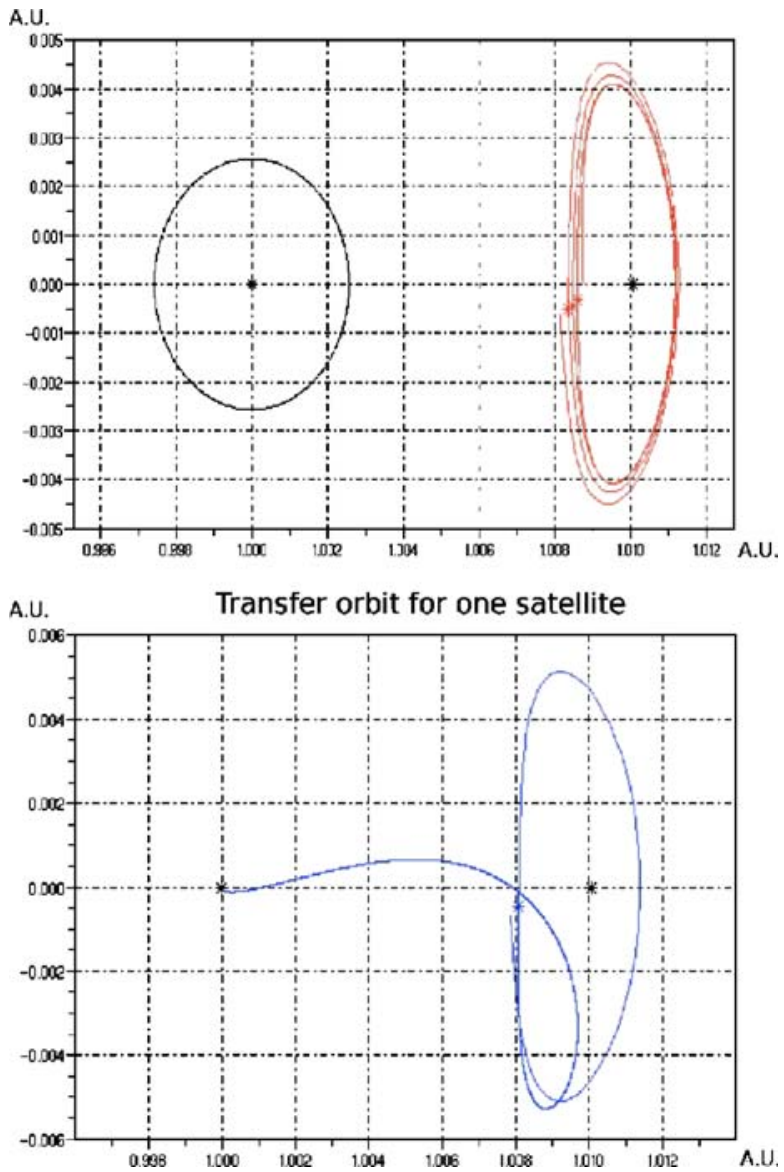

out of plane stable period of 184 days with amplitude of a few 10,000's km (Fig. 16a).

The pointing is anti-solar with $\mathrm{a} \pm 30^{\circ}$ allowed depointing angle. This gives access to more than $90 \%$ of the identified targets. The transfer is estimated to last about 120 days and the associated maneuvers require a $90 \mathrm{~m} / \mathrm{s} \Delta V$ for each spacecraft assuming they travel separately to L2. The station keeping requires very precise ranging every month, which is only possible with a deep space network.

\subsubsection{High elliptical orbit (HEO)}

An alternative solution is a High Elliptical Orbit (HEO). The gravity gradient is estimated compatible with the free flying requirements if the altitude is greater than $80,000 \mathrm{~km}$ (the residual forces are lower than the effects of the solar pressure). Several orbits with four to eight sideral days period, initial perigee from 20,000 to $44,000 \mathrm{~km}$ and initial apogee from 180,000 to $260,000 \mathrm{~km}$ 


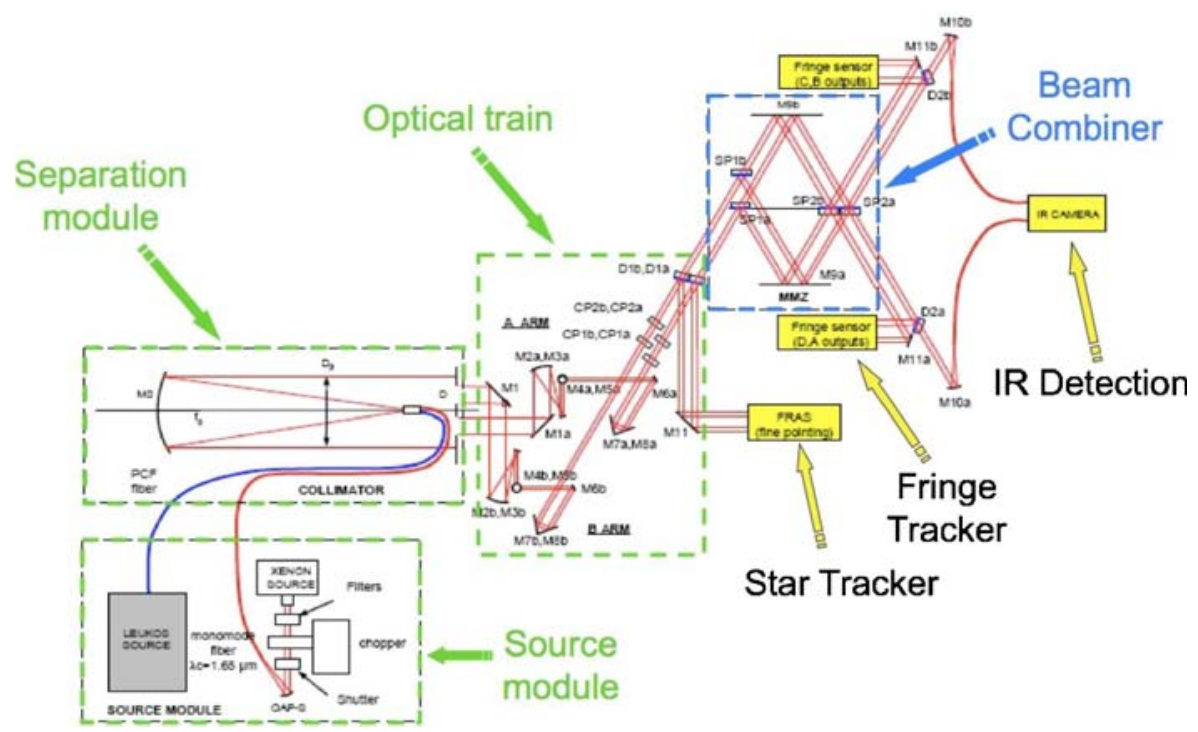

Fig. 17 Principle of PERSEE laboratory test bench

may be found to have more than $80 \%$ of time above this limit. The orbits with a too high apogee are quite unstable due to earth geo-potential tesseral terms and the moon attraction. Although the Soyuz performance is better for an HEO orbit than for an orbit around L2 (roughly 2,300 kg launched in this case, instead of $2,050 \mathrm{~kg}$ ), the need of a hydrazine propulsion module hampers the mass budget. Furthermore, when the position on the orbit and the season vary, the earth and moon apparent position change constantly which can lead to a more complex thermal design involving an earth shield or even a cryo-cooler.

\section{Key technology areas}

\subsection{Payload TRL ${ }^{2}$ level and technology development strategy}

The actual payload TRL level is 3 to 4 except the ODL, which reaches 5. CNES initiated a breadboard of the PEGASE payload, called PERSEE (PEGASE experiment for Research and Stabilization of Extreme Extinction, see Fig. 17). This 3 years R\&D activity performed in various French laboratories started in

\footnotetext{
${ }^{2}$ The Technology Readiness Level (TRL) qualifies the state of maturity of an instrumental concept, varying from 1: "Basic principle observed and reported" to 9: "Actual system "Flight proven" through successful mission operation". In order to be selected in 2007 by ESA, a Cosmic Vision proposal should be able to be considered in 2009 at TRL 6: "System/subsystem model or prototype demonstration in a relevant environment (ground or space)".
} 
2006. It will couple a nulling interferometer with fine control loops developed at ONERA and a GNC simulator developed by EADS-ASTRIUM or TAS (Thales Alenia Space). It shall demonstrate by the end of 2009 a $10^{-4}$ null at $10^{-5}$ stability over the range $1.65-3.3 \mu \mathrm{m}$ with full characterization of allowable external tip/tilt and opd perturbations. Most of the critical aspects will be covered: long term stability, coatings, polarization limits, monomode fibers, fringe acquisition bandpass, modes chaining (co-phasing, fringe tracking, scientific integration...). This work can greatly contribute to support a possible phase A assessment at payload and satellite levels.

The on ground testing of the payload is also a challenge. The integration will be performed at room temperature but the performances shall be demonstrated at $90 \mathrm{~K}$. The stability between these two temperatures is a serious issue, but many satellite builders have now a sufficient experience to provide adequate conception and testing for such cases.

\subsection{Mission and spacecraft technology challenges}

\subsubsection{Thrusters}

The MIB reduction down to $50 \mu \mathrm{N}$ s does not represent a strong technology push (a gain factor of 5 to 10 w.r.t. commercially available thruster's resolution). Starting by instance from a Marotta $10 \mathrm{mN}$ thrusters, the improvement seems affordable by reducing the minimal path of the thrusters divergent (today's diameter is $0.26 \mathrm{~mm}$ ), limiting the pulse duration and reducing the thrusters admission pressure (today it is roughly 1.3 bars for Marotta technology). The repeatability of the pulses would have to be studied in more details.

Another possibility is using a thruster control valve (Bradford Engineering). This product was developed for GOCE and is based on a AER solenoid valve. $0.5 \mathrm{mN}$ thrusts have been measured at Estec (ESA technical centre) in 2004, which would allow reaching a $0.01 \mathrm{mN}$ s MIB.

\subsubsection{GNC}

The first two modes will be fully demonstrated in flight by precursors missions like Prisma [17] and Proba 3 [5]. The critical part comes then from the maneuvers, the FDIR with three satellites, the fine control and the interface with the payload. The optimization of this interface and the good mitigation of the constraints is one of the key points of the mission.

\subsubsection{RF metrology}

The RF metrology is currently under development for the Swedish Prisma mission, which is planned to be launched in 2009. France participates in this program with a RF system and the associated GNC layer. The critical aspects of this kind of system will be tested in flight and this will provide the following free flying missions with a validated RF system and associated 
control. One particular aspect is the bias estimation difficulty due to possible multi-reflections on the satellites. One solution to solve this problem is that the optical sensor, required for the lateral control, can also provide a distance measurement at $\mathrm{cm}$ level with very few modifications. Another difficulty is the frequency band allocation and the compatibility with the telecommunication system. Last, the ISL (Inter Satellite Link) is a critical item for the formation control because it is used to exchange information between the satellites. Achieving the required reliability is a challenge.

\subsubsection{Optical sensor}

CNES and EADS-SODERN dedicated an R\&D program on that topic in the context of formation flying studies. The developed concept uses an adapted optical head with $3^{\circ} \mathrm{FOV}$ and an artificial target using a redundant fiber diode that generates 6 luminous points through fibers.

The sensor can perform lateral measurements with a static bias of 10 in., a thermal stability of 0.7 arcsec, a HF error of $0.5 \operatorname{arcsec}(3 \sigma)$ at $20 \mathrm{~m}$. Adding an auxiliary target at $0.7 \mathrm{~m}$ gives the possibility to measure the longitudinal distance with an error of $6 \mathrm{~mm}(3 \sigma)$ at $20 \mathrm{~m}$. Two types of diodes were selected and submitted to tests to evaluate their compatibility with space environment. They prove successful for both. The challenging point is now the reliability of these diodes that have to run continuously for years. The goal is to reach a 50,000 h MTBF.

\section{Conclusion}

At the end of the first "Cosmic Vision" process, PEGASE was not selected. The global strategy leading to an extrasolar planet direct detection mission will be reassessed. The PEGASE concept may thus be proposed again to ESA.

\section{References}

1. Absil, O., et al.: Circumstellar material in the Vega inner system revealed by CHARA/ FLUOR. A\&A 452, 237-244 (2006)

2. Allard, F., et al.: The limiting effect of dust in brown dwarf model atmosphere. ApJ. 556, 357372 (2001). doi:10.1086/321547

3. Barman, T.S., et al.: Irradiated planets. ApJ. 556, 885-895 (2001). doi:10.1086/321610

4. Beichman, C.A., et al: The Terrestrial Planet Finder: A NASA Origins Program to Search for Habitable Planets, pp. 99-3. JPL Publ. (1999)

5. Borde, J., et al.: Feasibility of the Proba 3 formation flying demonstration mission as a pair of microsats in GTO, ESA SP-571, p. 12.1 (2004)

6. Brachet, F.: Etude et développement d'un déphaseur achromatique pour l'interférométrie en frange noire. PhD report, University of Paris-Sud (XI) (2005)

7. Bracewell, R.N.: Detecting non solar planets by spinning infrared interferometer. Nature 274, 780 (1978). doi:10.1038/274780a0

8. Cassaing, F., et al.: An optimized fringe tracker for the VLTI/PRIMA instrument. Proc SPIE 4006, 152-163 (2000) 
9. Claeskens, J.-F., Surdej, J.: Gravitational lensing in Quasar samples. A\&A Rev. 10, 263-311 (2002)

10. Di Folco, E., et al.: A near-IR interferometric survey of debris-disk stars I. Probing the hot dust content around epsilon Eri and Tau Cet with CHARA/FLUOR. A\&A 475, 243-250 (2007)

11. Fridlund, M., et al.: DARWIN, the Infrared Space Interferometer. ESA-SCI(2000)12 (2000)

12. Gabor, P., et al.: Stabilizing a nulling interferometer using optical path difference dithering. A\&A 483, 365-369 (2008)

13. Le Duigou, J.M., et al.: PEGASE: a space-based nulling interferometer. Proc. SPIE 6265, 47 (2006)

14. Mann, I., et al.: Dust in the solar system and in extra-solar planetary systems. A\&A Rev. 13, 159-228 (2006)

15. Mennesson, B., Mariotti, J.-M.: Array configurations for a space infrared nulling interferometer dedicated to the search for earthlike extrasolar planets. Icarus 128, 202-212 (1997). doi:10.1006/icar.1997.5731

16. Meyer, M.R., et al.: The formation and evolution of planetary systems: placing our solar system in context with spitzer. PASP 118, 1690-1710 (2007)

17. Persson, S., et al.: PRISMA-Demonstration Mission for Advanced Rendezvous and Formation Flying Technologies and Sensors. 56th International Astronautical Congress (IAF), Fukuoka, Japan (2005)

18. Serabyn, E., Colavita, M.M.: Fully symmetric nulling beam combiners. Appl. Opt. 40, 16681671 (2001). doi:10.1364/AO.40.001668

19. Shao, M., et al.: The Mark III stellar interferometer. A\&A 193, 357-371 (1988)

20. Sudarsky, D., et al.: Theoretical spectra and atmospheres of extrasolar giant planets. ApJ. 588, 1121-1148 (2003). doi:10.1086/374331

21. Van den Dool T., et al.: The Darwin Breadboard cryogenic optical delay line Darwin. ICSO 2006 conference, Noordwijk (June 2006)

22. Villien, A., et al.: GNC for the PEGASE mission. 17th IFAC Symposium on Automatic Control in Aerospace, Toulouse (June 2007)

23. Weber, V., et al.: Nulling interferometer based on an integrated optics combiner. Proc. SPIE 5491, 842 (2004). doi:10.1117/12.550581 